\title{
MULTITYPE SHAPE THEOREMS FOR FIRST PASSAGE PERCOLATION MODELS
}

\author{
LEANDRO P. R. PIMENTEL, * École Polytechnique Fédérale de Lausanne
}

\begin{abstract}
A Euclidean first passage percolation model describing the competing growth between $k$ different types of infection is considered. We focus on the long-time behavior of this multitype growth process and we derive multitype shape results related to its morphology.

Keywords: First passage percolation; competing growth; multitype shape theorem; competition interface
\end{abstract}

2000 Mathematics Subject Classification: Primary 60K35

Secondary 82B43; 60D05

\section{Introduction}

In standard planar first passage percolation [9], each pair $\boldsymbol{x}$ and $\boldsymbol{y}$ of nearest neighbors in $\mathbb{Z}^{2}$ has an edge connecting them and each edge is equipped with a nonnegative random variable (the passage time) which may be interpreted as the time it takes for an infection to be transmitted from $\boldsymbol{x}$ to $\boldsymbol{y}$. We assume that these random variables are independent and identically distributed with a continuous distribution $\mathbb{F}$. The passage time, $t(\gamma)$, for a nearest-neighbor path $\gamma$ is simply the sum of the passage times along the path. For $\boldsymbol{x}, \boldsymbol{y} \in \mathbb{Z}^{2}$, the first passage time from $\boldsymbol{x}$ to $\boldsymbol{y}$, which we denote by $T(\boldsymbol{x}, \boldsymbol{y})$, is the infimum of $t(\gamma)$ over all paths $\gamma$ from $\boldsymbol{x}$ to $\boldsymbol{y}$. For $t \geq 0$, let $\boldsymbol{B}(t)$ be the set of sites $\boldsymbol{x}$ reached from the origin, $\mathbf{0}$, by time $t$, i.e. for which $T(\boldsymbol{0}, \boldsymbol{x}) \leq t$. One may think of sites in $\boldsymbol{B}(t)$ as being infected and those in $\boldsymbol{B}(t)^{\mathrm{c}}$ as being healthy, and of the origin as being infected by some type of disease at time 0 . The process $\{\boldsymbol{B}(t): t \geq 0\}$ is then a model for the growth of an infection.

An interesting aspect of the evolution of the infection, namely the tree of infection, is constructed as follows. First notice that, since the passage time distribution is continuous, for all $\boldsymbol{x}, \boldsymbol{y} \in \mathbb{Z}^{2}$ there is (almost surely) a unique time-minimizing path (or geodesic) from $\boldsymbol{x}$ to $\boldsymbol{y}$, which we denote by $\rho(\boldsymbol{x}, \boldsymbol{y})$, such that $T(\boldsymbol{x}, \boldsymbol{y})=t(\rho(\boldsymbol{x}, \boldsymbol{y}))$. Thus, $\rho(\boldsymbol{x}, \boldsymbol{y})$ may be interpreted as the path through which the infection was transmitted from $\boldsymbol{x}$ to $\boldsymbol{y}$. With this picture in mind, the tree of infection, $\Gamma$, is defined by the union over all $\boldsymbol{x} \in \mathbb{Z}^{2}$ of the edges $\boldsymbol{e} \in \rho(\mathbf{0}, \boldsymbol{x})$. Newman [14] has shown that the number, $K(\Gamma)$, of topological ends of $\Gamma$, i.e. the number of semi-infinite self-avoiding paths in $\Gamma$, is infinite provided there exist an exponential moment condition on $\mathbb{F}$ and a certain hypothesis concerning the uniformly bounded curvature of the asymptotic shape of $\boldsymbol{B}(t)$. Although the curvature hypothesis is plausible, it has so far not been proved.

In order to study the tree of infection, Häggström and Pemantle [7], [8] have introduced the following multitype growth model. At time 0 we start with $k$ different sites of $\mathbb{Z}^{2}$, say $\boldsymbol{x}_{1}, \ldots, \boldsymbol{x}_{k}$, each one representing a different type of infection. A site $\boldsymbol{y} \in \mathbb{Z}^{2}$ is then infected at

Received 8 February 2006; revision received 31 October 2006.

* Postal address: Institut de Mathématiques, École Polytechnique Fédérale de Lausanne, CH-1015 Lausanne, Switzerland. Email address: leandro.pimentel@epfl.ch 


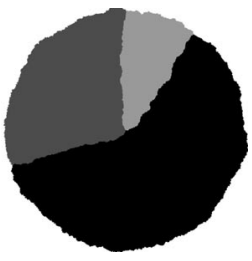

(a)

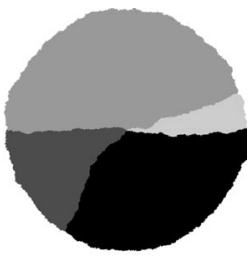

(b)

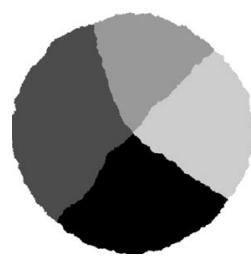

(c)

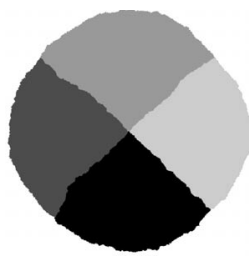

(d)

FIGURE 1: We performed numerical simulations for the multitype first passage percolation model on $\mathbb{Z}^{2}$ with (mean-1) exponential passage times. We chose there to be four different species $(k=4)$, starting from the initial conditions $\boldsymbol{x}_{1}(r)=(r, 0)$ (light gray), $\boldsymbol{x}_{2}(r)=(0, r)$ (medium gray), $\boldsymbol{x}_{3}(r)=(-r, 0)$ (dark gray), and $\boldsymbol{x}_{4}(r)=(0,-r)$ (black). These pictures show the result at time $t=200$ for (a) $r=1$, (b) $r=3$, (c) $r=50$, and (d) $r=100$. Notice that in (a) type 1 (light gray) has not survived, while in

(d) each type has conquered almost the same volume.

time $\min \left\{T\left(\boldsymbol{x}_{1}, \boldsymbol{y}\right), \ldots, T\left(\boldsymbol{x}_{k}, \boldsymbol{y}\right)\right\}$, by the infection which first arrives there, i.e. by the unique type $j \in\{1, \ldots, k\}$ such that $T\left(\boldsymbol{x}_{j}, \boldsymbol{y}\right)=\min \left\{T\left(\boldsymbol{x}_{1}, \boldsymbol{y}\right), \ldots, T\left(\boldsymbol{x}_{k}, \boldsymbol{y}\right)\right\}$ (see Figure 1). It may happen that at some early stage one of the types of infection completely surrounds another one, which then is prevented from growing indefinitely. If this does not occur or, equivalently, if all types of infection grow unboundedly, we say that $k$-coexistence occurs.

Returning to the subject of the topological ends of $\Gamma$, Häggström and Pemantle [7] have noted that if $k$-coexistence occurs with positive probability, then $K(\Gamma) \geq k$ with positive probability. They have also shown that if one considers an exponential passage time distribution, then 2-coexistence occurs with positive probability and, thus, $K(\Gamma) \geq 2$ with positive probability. Later, Garet and Marchand [6] and Hoffman [10] extended this last result to stationary and ergodic first passage percolation models on $\mathbb{Z}^{d}$.

In this work we focus on the long-time behavior of this multitype growth model. However, unlike the above-mentioned authors, we choose a first passage percolation setup on a random Delaunay triangulation [20] whose spherical symmetry (isotropy) ensures that the asymptotic shape of the corresponding growth process is a Euclidean ball. This choice allows us, by following Newman [14], to prove various statements concerning path minimization, such as Propositions 4.1, 4.2, and 4.3 (see also [11] and [12] for other Euclidean first passage percolation models). In this setting, the main results we will prove are as follows.

- If a type of infection survives then the region it conquers is (asymptotically) a cone with a random angle (see Theorem 1.1 and Remark 1.1).

- If the $k$ initial sites form a regular polygon of radius $r$ centered at the origin, then the probability that $k$-coexistence occurs tends to 1 as $r$ tends to $\infty$. Moreover, for all $\varepsilon>0$, the probability that for all $j \in\{1, \ldots, k\}$ the region conquered by infection $j$ contains (asymptotically) the cone with axis through $\mathbf{0}$ and $\boldsymbol{x}_{j}$ and angle $\pi / k-\varepsilon$ also tends to 1 (see Theorem 1.2).

To prove our results, the main idea is to explore the relation between this multitype growth model and the asymptotic behavior of $T\left(\boldsymbol{x}, \boldsymbol{y}_{n}\right)-T\left(\mathbf{0}, \boldsymbol{y}_{n}\right)$ as $\boldsymbol{y}_{n}$ goes to $\infty$ along a ray of angle $\alpha$ (see Theorems 1.3 and 1.4). We also study some roughening aspects of the onedimensional boundary between the infections (the competition interface) which were pointed out by physicists in numerical simulations [3], [19] (see Remark 1.1). We note that analogous problems in the context of last passage percolation and totally asymmetric exclusion processes 
were treated by Ferrari and Pimentel [4] and Ferrari et al. [5]. Also, Deijfen et al. [2] have considered isotropic multitype growth models in $\mathbb{R}^{d}$ where the growth is driven by outbursts in the infected region.

\subsection{The multitype growth process}

Consider the random graph $\mathscr{D}:=\left(\mathscr{D}_{\mathrm{v}}, \mathscr{D}_{\mathrm{e}}\right)$, named the Delaunay triangulation, constructed as follows. The vertex set, $\mathscr{D}_{\mathrm{v}} \subseteq \mathbb{R}^{2}$, is the set of points realized in a two-dimensional homogeneous Poisson point process with intensity 1 . To each vertex $v$ corresponds an open and bounded polygonal region $\boldsymbol{C}_{\boldsymbol{v}}$ (the Voronoi tile at $\boldsymbol{v}$ ) consisting of the set of points in $\mathbb{R}^{2}$ which are closer to $\boldsymbol{v}$ than to any other vertex $\boldsymbol{v}^{\prime} \in \mathscr{D}_{\mathrm{v}}$. The edge set, $\mathscr{D}_{\mathrm{e}}$, consists of nonoriented pairs $\left(\boldsymbol{v}, \boldsymbol{v}^{\prime}\right)$ such that $\boldsymbol{C}_{\boldsymbol{v}}$ and $\boldsymbol{C}_{\boldsymbol{v}^{\prime}}$ share a one-dimensional edge (see Figure 2). One can see that (with probability 1 ) each Voronoi tile is a convex and bounded polygon, and the graph $\mathcal{D}:=\left(\mathscr{D}_{\mathrm{v}}, \mathscr{D}_{\mathrm{e}}\right)$ is a triangulation of the plane. The Voronoi tessellation, $\mathcal{V}:=\left(\mathcal{V}_{\mathrm{v}}, \mathcal{V}_{\mathrm{e}}\right)$, is defined by choosing the vertex set $\mathcal{V}_{\mathrm{v}}$ to equal the set of vertices of the Voronoi tiles and the edge set $\mathcal{V}_{\mathrm{e}}$ to equal the set of edges of the Voronoi tiles.

Each edge $\boldsymbol{e} \in \mathscr{D}_{\mathrm{e}}$ is independently assigned a nonnegative random variable $\tau_{\boldsymbol{e}}$ (the passage time) from a common distribution $\mathbb{F}$ (the passage time distribution) that is independent of the Poisson process $\mathscr{D}_{\mathrm{v}}$. We assume throughout that $\mathbb{F}$ is continuous and that

$$
\int \mathrm{e}^{a x} \mathbb{F}(\mathrm{d} x)<\infty \quad \text { for some } a \in(0, \infty)
$$

We denote by $(\Omega, \mathcal{F}, \mathrm{P})$ our underlying probability space: from each realization $\omega \in \Omega$, we can determine the Poisson point process as well as the passage time configuration. This model inherits the Euclidean (translation and rotation) invariance of the Poisson point process.

The passage time, $t(\gamma)$, of a path $\gamma$ in $\mathscr{D}$ is the sum of the passage times of the edges in $\gamma$ :

$$
t(\gamma):=\sum_{\boldsymbol{e} \in \gamma} \tau_{\boldsymbol{e}}
$$

The first passage time between two vertices $\boldsymbol{v}$ and $\boldsymbol{v}^{\prime}$ in $\mathscr{D}_{\mathrm{v}}$ is defined by

$$
T\left(\boldsymbol{v}, \boldsymbol{v}^{\prime}\right):=\inf \left\{t(\gamma): \gamma \in \mathcal{C}\left(\boldsymbol{v}, \boldsymbol{v}^{\prime}\right)\right\}
$$

where $\mathcal{C}\left(\boldsymbol{v}, \boldsymbol{v}^{\prime}\right)$ is the set of all paths connecting $\boldsymbol{v}$ and $\boldsymbol{v}^{\prime}$. We extend the definition of the first passage time $T$ to $\boldsymbol{x}, \boldsymbol{y} \in \mathbb{R}^{2}$ by setting $T(\boldsymbol{x}, \boldsymbol{y}):=T(\boldsymbol{v}(\boldsymbol{x}), \boldsymbol{v}(\boldsymbol{y}))$, where $\boldsymbol{v}(\boldsymbol{x})$ is the almost

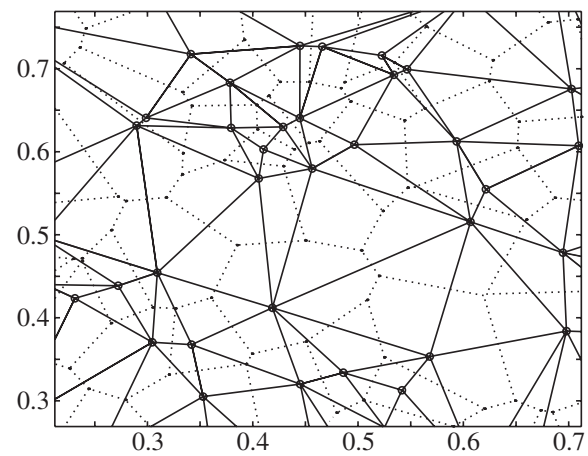

FIGURE 2: The Delaunay triangulation (solid lines) and the Voronoi tessellation (dashed lines). 
surely unique vertex $\boldsymbol{v} \in \mathcal{P}$ with $\boldsymbol{x} \in \boldsymbol{C}_{\boldsymbol{v}}$. We say that $\rho\left(\boldsymbol{v}, \boldsymbol{v}^{\prime}\right) \in \mathcal{C}\left(\boldsymbol{v}, \boldsymbol{v}^{\prime}\right)$ is a geodesic between $\boldsymbol{v}$ and $\boldsymbol{v}^{\prime}$ if $t\left(\rho\left(\boldsymbol{v}, \boldsymbol{v}^{\prime}\right)\right)=T\left(\boldsymbol{v}, \boldsymbol{v}^{\prime}\right)$. For each $\boldsymbol{x}, \boldsymbol{y} \in \mathbb{R}^{2}$ we let $\rho(\boldsymbol{x}, \boldsymbol{y}):=\rho(\boldsymbol{v}(\boldsymbol{x}), \boldsymbol{v}(\boldsymbol{y}))$. We see that if $\mathbb{F}$ is a continuous function, then for all $\boldsymbol{v}, \boldsymbol{v}^{\prime} \in \mathscr{D}_{\mathrm{v}}$ there almost surely exists a unique geodesic $\rho\left(\boldsymbol{v}, \boldsymbol{v}^{\prime}\right)$ [18]. A self-avoiding and semi-infinite path $\rho=\left(\boldsymbol{v}_{1}, \boldsymbol{v}_{2}, \ldots\right)$ in $\mathcal{D}$ is called a semi-infinite geodesic if, for all $\boldsymbol{v}_{j}, \boldsymbol{v}_{k} \in \rho$, the path $\left(\boldsymbol{v}_{j}, \boldsymbol{v}_{j+1}, \ldots, \boldsymbol{v}_{k}\right)$ is the unique geodesic connecting $\boldsymbol{v}_{j}$ and $\boldsymbol{v}_{k}$.

Given $k$ different points $\boldsymbol{x}_{1}, \ldots, \boldsymbol{x}_{k} \in \mathbb{R}^{2}$, the initial configuration of seeds, we define the multitype growth process $\left\{\left(\boldsymbol{B}_{\boldsymbol{x}_{1}}(t), \ldots, \boldsymbol{B}_{\boldsymbol{x}_{k}}(t)\right): t \geq 0\right\}$ with

$$
\boldsymbol{B}_{\boldsymbol{x}_{j}}(t):=\left\{\boldsymbol{x} \in \mathbb{R}^{2}: \boldsymbol{x} \in c\left(\boldsymbol{C}_{\boldsymbol{v}}\right) \text { for some } \boldsymbol{v} \in \mathcal{B}_{\boldsymbol{x}_{j}}(t)\right\},
$$

where

$$
\mathscr{B}_{\boldsymbol{x}_{j}}(t):=\left\{\boldsymbol{v} \in \mathscr{D}_{\mathrm{v}}: T\left(\boldsymbol{x}_{j}, \boldsymbol{v}\right) \leq t \text { and } \min _{l=1, \ldots, k}\left\{T\left(\boldsymbol{x}_{l}, \boldsymbol{v}\right)\right\}=T\left(\boldsymbol{x}_{j}, \boldsymbol{v}\right)\right\}
$$

and $c\left(\boldsymbol{C}_{\boldsymbol{v}}\right)$ denotes the closure of the tile $\boldsymbol{C}_{\boldsymbol{v}}$. If there exists a $j<l$ such that $\boldsymbol{v}_{\boldsymbol{x}_{j}}=\boldsymbol{v}_{\boldsymbol{x}_{l}}$, then we set $\boldsymbol{B}_{\boldsymbol{x}_{j}}$ as before and $\boldsymbol{B}_{\boldsymbol{x}_{l}}(t)=\varnothing$.

If $k=1$ then we have a single growth process, $\boldsymbol{B}_{\boldsymbol{x}}(t)$, which represents the set of points reached by time $t$ starting from the initial seed $\boldsymbol{x}$. For a continuous distribution $\mathbb{F}$ satisfying (1.1), the following shape theorem [18], [21] holds: there exists a constant $\mu(\mathbb{F}) \in(0, \infty)$, namely the time constant, such that, for all $\varepsilon>0$,

$$
\mathrm{P}\left((1-\varepsilon) t \boldsymbol{D}(1 / \mu) \subseteq \boldsymbol{B}_{\mathbf{0}}(t) \subseteq(1+\varepsilon) t \boldsymbol{D}(1 / \mu) \text { eventually }\right)=1,
$$

where $\boldsymbol{D}(r):=\left\{\boldsymbol{x} \in \mathbb{R}^{2}:|\boldsymbol{x}| \leq r\right\}$ and $\mathbf{0}:=(0,0)$.

For $k \geq 2$, the process $\left\{\left(\boldsymbol{B}_{\boldsymbol{x}_{1}}(t), \ldots, \boldsymbol{B}_{\boldsymbol{x}_{k}}(t)\right): t \geq 0\right\}$ is a model for competing growth on the plane where each point $\boldsymbol{x} \in \mathbb{R}^{2}$ is acquired by the species $j \in\{1, \ldots, k\}$ which first arrives there. The competition interface, $\psi$, is the one-dimensional boundary between the species when $t=\infty$. This interface can be seen as a finite union of polygonal curves determined by edges in $\mathcal{V}$ (the Voronoi tessellation) which are shared by tiles acquired by different species. A branch of the competition interface is a self-avoiding path $\varphi=\left(\boldsymbol{x}_{n}\right)_{n \geq 1}$ in $\mathcal{V}$ such that $\left\{\boldsymbol{x}_{n}: n \geq 1\right\} \subseteq \psi$.

For each $\alpha \in[0,2 \pi)$, we say that a self-avoiding path $\left(\boldsymbol{x}_{n}\right)_{n \geq 1}$, with vertices in $\mathbb{R}^{2}$ and such that $\left|\boldsymbol{x}_{n}\right| \rightarrow \infty$ when $n \rightarrow \infty$, is an $\alpha$-path if

$$
\lim _{n \rightarrow \infty} \frac{\boldsymbol{x}_{n}}{\left|\boldsymbol{x}_{n}\right|}=\mathrm{e}^{\mathrm{i} \alpha}:=(\cos \alpha, \sin \alpha)
$$

In this case we also say that $\left(\boldsymbol{x}_{n}\right)_{n \geq 1}$ has the asymptotic orientation $\mathrm{e}^{\mathrm{i} \alpha}$. This is equivalent to

$$
\lim _{n \rightarrow \infty} \operatorname{ang}\left(\boldsymbol{x}_{n}, \mathrm{e}^{\mathrm{i} \alpha}\right)=0,
$$

where ang $(\boldsymbol{x}, \boldsymbol{y})$ denotes the angle in $[0, \pi]$ between the points $\boldsymbol{x}, \boldsymbol{y} \in \mathbb{R}^{2}$, measured at the origin. Thus, a sufficient condition for a path $\left(\boldsymbol{x}_{n}\right)_{n \geq 1}$ to be an $\alpha$-path for some $\alpha \in[0,2 \pi)$ is that, for some fixed $\delta \in(0,1)$, some constant $c>0$, and sufficiently large $n$,

$$
\operatorname{ang}\left(\boldsymbol{x}_{n}, \boldsymbol{x}_{m}\right) \leq\left|\boldsymbol{x}_{n}\right|^{-\delta} \text { for } m>n \text {. }
$$

This is the $\delta$-straightness property for semi-infinite paths, introduced by Newman [14]. 
Theorem 1.1. For $k \geq 2$, let $\Omega_{k}$ be the event that, for the competing growth model with $k$ different species, there exists a finite subset $\Theta:=\left\{\theta_{1}, \ldots, \theta_{m}\right\}$ of $[0,2 \pi)$ such that every branch $\varphi$ of the competition interface is a $\theta_{i}(\varphi)$-path for some $\theta_{i} \in \Theta$. Under (1.1), $\mathrm{P}\left(\Omega_{k}\right)=1$.

Remark 1.1. In Section 4.4 we will give a sketch of the proof that, for all $\alpha \in[0,2 \pi)$,

$$
\mathrm{P}\left(\theta_{i}=\alpha \text { for some } \theta_{i} \in \Theta\right)=0,
$$

and that if $\xi \in\left(\frac{3}{4}, 1\right)$ then, for all branches $\varphi=\left(\boldsymbol{x}_{n}\right)_{n \geq 1}$ of the competition interface, there almost surely exists a constant $c>0$ such that

$$
\operatorname{ang}\left(\boldsymbol{x}_{n}, \mathrm{e}^{\mathrm{i} \theta(\varphi)}\right) \leq c\left|\boldsymbol{x}_{n}\right|^{\xi-1} \quad \text { eventually. }
$$

Let $\boldsymbol{x}_{1}(r)=(0, r), \ldots, \boldsymbol{x}_{k}(r)$ be the vertices of a regular polygon with $k$ sides and radius $r$. For each $j=1, \ldots, k$, define the projection of the random set $\boldsymbol{B}_{j}^{r}:=\boldsymbol{B}_{\boldsymbol{x}_{j}(r)}(\infty)$ onto $\boldsymbol{S}^{1}$, the set of unit vectors, by

$$
S_{j, r}:=\left\{\boldsymbol{x}=\mathrm{e}^{\mathrm{i} \alpha} \in \boldsymbol{S}^{1}: \mathbb{L}_{s \boldsymbol{x}}(\alpha) \subseteq \boldsymbol{B}_{j}^{r} \text { for some } s>0\right\},
$$

where $\mathbb{L}_{\boldsymbol{x}}(\alpha)$ denotes the line with origin $\boldsymbol{x}$ and direction $\mathrm{e}^{\mathrm{i} \alpha}$. For each $\varepsilon \in(0, \pi / k)$ and $j \in\{1, \ldots, k\}$, define

$$
S_{j}(\varepsilon):=\left\{x \in S^{1}: \operatorname{ang}\left(x, x_{j}(r)\right) \leq \pi / k-\varepsilon\right\} .
$$

Theorem 1.2. Let $k \geq 2$. Under (1.1), for all $\varepsilon>0$,

$$
\lim _{n \rightarrow \infty} \mathrm{P}\left(\boldsymbol{S}_{j}(\varepsilon) \subseteq \boldsymbol{S}_{j, n} \text { for all } j=1, \ldots, k\right)=1 .
$$

\subsection{Busemann-type asymptotics and the competition interface}

To illustrate the approach we follow in this work to study the competition interface, assume that $k=2$. Consider the line, $\mathbb{L}_{\mathbf{0}}(\alpha)$, with origin $\mathbf{0}$ and direction $\mathrm{e}^{\mathrm{i} \alpha}$. Then we have three possibilities: (i) it intersects the competition interface infinitely many times; (ii) it is eventually contained in $\boldsymbol{B}_{\boldsymbol{x}_{1}}(\infty)$; or (iii) it is eventually contained in $\boldsymbol{B}_{\boldsymbol{x}_{2}}(\infty)$. Notice that option (i) implies that

$$
\liminf _{s \rightarrow \infty}\left(T\left(\boldsymbol{x}_{1}, s \mathrm{e}^{\mathrm{i} \alpha}\right)-T\left(\boldsymbol{x}_{2}, s \mathrm{e}^{\mathrm{i} \alpha}\right)\right) \leq 0 \leq \limsup _{s \rightarrow \infty}\left(T\left(\boldsymbol{x}_{1}, s \mathrm{e}^{\mathrm{i} \alpha}\right)-T\left(\boldsymbol{x}_{2}, s \mathrm{e}^{\mathrm{i} \alpha}\right)\right),
$$

option (ii) implies that

$$
\limsup _{s \rightarrow \infty}\left(T\left(\boldsymbol{x}_{1}, s \mathrm{e}^{\mathrm{i} \alpha}\right)-T\left(\boldsymbol{x}_{2}, s \mathrm{e}^{\mathrm{i} \alpha}\right)\right) \leq 0,
$$

and option (iii) implies that

$$
0 \leq \liminf _{s \rightarrow \infty}\left(T\left(\boldsymbol{x}_{1}, s \mathrm{e}^{\mathrm{i} \alpha}\right)-T\left(\boldsymbol{x}_{2}, s \mathrm{e}^{\mathrm{i} \alpha}\right)\right) .
$$

It turns out that the above expressions resemble Busemann-type asymptotics for $T$. Newman [14] and Licea and Newman [13] have shown for the lattice model that, under suitable assumptions on the curvature of the limit shape, $T\left(\boldsymbol{x}_{1}, \boldsymbol{y}_{n}\right)-T\left(\boldsymbol{x}_{2}, \boldsymbol{y}_{n}\right)$ eventually attains a nonzero value $H^{\alpha}\left(\boldsymbol{x}_{1}, \boldsymbol{x}_{2}\right)$, called the Busemann function. By following Newman's method, and by exploiting the isotropy in our model, we will prove the following result. 
Theorem 1.3. For $\alpha \in[0,2 \pi)$, let $\Omega_{0}(\alpha)$ be the event that for all $\boldsymbol{v}, \overline{\boldsymbol{v}} \in \mathscr{D}_{\mathrm{v}}$ there exists an $H^{\alpha}(\boldsymbol{v}, \overline{\boldsymbol{v}})$, nonzero for $\boldsymbol{v} \neq \overline{\boldsymbol{v}}$, such that

$$
\lim _{\substack{|x| \rightarrow \infty \\ x /|x| \rightarrow \mathrm{e}^{\mathrm{i} \alpha}}}(T(\boldsymbol{v}, \boldsymbol{x})-T(\overline{\boldsymbol{v}}, \boldsymbol{x}))=H^{\alpha}(\boldsymbol{v}, \overline{\boldsymbol{v}}) .
$$

Under (1.1), $\mathrm{P}\left(\Omega_{0}(\alpha)\right)=1$.

(We also refer the reader to [11], where an analogous result was proved in a Euclidean first passage percolation setup.)

For $\boldsymbol{x}, \boldsymbol{y} \in \mathbb{R}^{2}$, we let $H^{\alpha}(\boldsymbol{x}, \boldsymbol{y}):=H^{\alpha}(\boldsymbol{v}(\boldsymbol{x}), \boldsymbol{v}(\boldsymbol{y}))$. It was conjectured by Howard and Newman [12] that

$$
\lim _{n \rightarrow \infty} \frac{H^{\alpha}\left(n \vec{e}_{1}, \mathbf{0}\right)}{n}=-\mu(\mathbb{F}) \cos \alpha,
$$

where $\vec{e}_{1}:=(1,0)$. This observation is related to the asymptotic behavior of our multitype growth model and the result key in proving Theorem 1.2 is the following, which is a small step towards the above conjecture.

Theorem 1.4. For $\alpha \in[0, \pi / 2)$, let $\Omega_{1}(\alpha) \subseteq \Omega_{0}(\alpha)$ be the event that

$$
-\mu(\mathbb{F}) \leq \liminf _{n \rightarrow \infty} \frac{H^{\alpha}\left(n \vec{e}_{1}, \mathbf{0}\right)}{n} \leq \limsup _{n \rightarrow \infty} \frac{H^{\alpha}\left(n \vec{e}_{1}, \mathbf{0}\right)}{n} \leq-\mu(\mathbb{F}) \frac{\cos \alpha}{1+\sin \alpha} .
$$

Under (1.1), $\mathrm{P}\left(\Omega_{1}(\alpha)\right)=1$. In particular, with probability 1 ,

$$
\lim _{n \rightarrow \infty} \frac{H^{0}\left(n \vec{e}_{1}, \mathbf{0}\right)}{n}=-\mu(\mathbb{F}) .
$$

\subsection{Overview}

In Section 2 we will deduce Theorems 1.1 and 1.2 from Theorems 1.3 and 1.4. In Section 3 we will start by defining the probability space underlying our model, and we will prove a 'modification lemma' that will play an important rule in the study of the coalescence of semiinfinite geodesics. Then we will study some geometrical aspects of Voronoi tilings. We note that in the Delaunay triangulation context some technical difficulties are imposed by its long-range dependence. Some of them will be avoided by appealing to results of previous work of the author [18], [17]. To conclude Section 3 we will recall some geometrical lemmas concerning the $\delta$-straightness of semi-infinite paths. Finally, in Section 4 we will study the existence and coalescence of semi-infinite geodesics to prove Theorems 1.3 and 1.4. Our treatment will largely parallel the analogous study on the lattice and in the Euclidean first passage percolation models that was developed by Newman and various co-authors [12], [13], [14], [15].

\section{Proof of the multitype shape theorems}

Proof of Theorem 1.1. For each $j=1, \ldots, k$, let $\boldsymbol{S}_{j}$ denote the set of unit vectors $\mathrm{e}^{\mathrm{i} \beta}$ such that $\mathbb{L}_{s \mathrm{e}^{\mathrm{i} \beta}}(\beta) \subseteq \boldsymbol{B}_{\boldsymbol{x}_{j}}(\infty)$ for some $s>0$, and let

$$
S_{0}:=\left(\bigcup_{j=1}^{l} S_{j}\right)^{\mathrm{c}}
$$


Let

$$
\boldsymbol{D}_{n}:=\left\{\mathrm{e}^{\mathrm{i} \beta}: \beta=2 k \pi / 2^{n} \text { for some } k, 1 \leq k \leq 2^{n}\right\}
$$

and $\boldsymbol{D}:=\bigcup_{n \geq 1} \boldsymbol{D}_{n}$. Consider the event, $\bigcap_{\alpha \in \boldsymbol{D}} \Omega_{0}(\alpha)$, that for all $\alpha \in \boldsymbol{D}$ and $\boldsymbol{v}, \overline{\boldsymbol{v}} \in \mathscr{D}_{\mathrm{v}}$ there exists an $H^{\alpha}(\bar{v}, \bar{v})$, nonzero for $\boldsymbol{v} \neq \overline{\boldsymbol{v}}$, such that

$$
\lim _{\substack{|\boldsymbol{x}| \rightarrow \infty \\ \boldsymbol{x} /|\boldsymbol{x}| \rightarrow \mathrm{e}^{\mathrm{i} \alpha}}}(T(\boldsymbol{v}, \boldsymbol{x})-T(\overline{\boldsymbol{v}}, \boldsymbol{x}))=H^{\alpha}(\boldsymbol{v}, \overline{\boldsymbol{v}}) .
$$

By Theorem 1.3, $\mathrm{P}\left(\bigcap_{\alpha \in D} \Omega_{0}(\alpha)\right)=1$.

We claim that, on this event, every branch of the competition interface is a $\theta$-path for some $\theta \in[0,2 \pi)$. To see this, note that if $\mathrm{e}^{\mathrm{i} \alpha} \in \boldsymbol{S}_{0}$ then, for some $j_{1} \neq j_{2}, \mathbb{L}_{\mathbf{0}}(\alpha)$ intersects the region $\boldsymbol{B}_{\boldsymbol{x}_{j_{1}}}(\infty)$ and the region $\boldsymbol{B}_{\boldsymbol{x}_{j_{2}}}$ infinitely many times. Thus,

$$
\liminf _{s \rightarrow \infty}\left(T\left(\boldsymbol{x}_{j_{1}}, s \mathrm{e}^{\mathrm{i} \alpha}\right)-T\left(\boldsymbol{x}_{j_{2}}, s \mathrm{e}^{\mathrm{i} \alpha}\right)\right) \leq 0 \leq \limsup _{s \rightarrow \infty}\left(T\left(\boldsymbol{x}_{j_{1}}, s \mathrm{e}^{\mathrm{i} \alpha}\right)-T\left(\boldsymbol{x}_{j_{2}}, s \mathrm{e}^{\mathrm{i} \alpha}\right)\right),
$$

which implies that $\boldsymbol{D} \cap \boldsymbol{S}_{0}=\varnothing$. Let $\boldsymbol{C}_{k}^{n}$ be the cone consisting of the points $r \mathrm{e}^{\mathrm{i} \beta}$ such that $r>0$ and $\beta \in\left(2 \pi k / 2^{n}, 2 \pi(k+1) / 2^{n}\right)$. Now, if $\boldsymbol{D} \cap \boldsymbol{S}_{0}=\varnothing$ and $\mathrm{e}^{\mathrm{i} \beta} \in \boldsymbol{D}$, then no branch of the competition interface can intersect the line $\mathbb{L}_{\mathbf{0}}(\beta)$ infinitely many times. Hence, for each branch $\varphi$ of the competition interface we can find a sequence of cones $\left(\boldsymbol{C}_{k_{n}}^{n}\right)_{n \geq 1}$, with $n \rightarrow \infty$ and $\boldsymbol{C}_{k_{n+1}}^{n+1} \subseteq \boldsymbol{C}_{k_{n}}^{n}$, such that $\varphi$ is eventually contained in $\boldsymbol{C}_{k_{n}}^{n}$. Note that the angle of the cone $\boldsymbol{C}_{k_{n}}^{n}$ is $2^{n}$ and that the sequence $\left(\boldsymbol{C}_{k_{n}}^{n}\right)$ thus converges to a semi-infinite line starting at the origin, $\mathbf{0}$. This implies that $\varphi$ must be a $\theta$-path for some $\theta \in[0,2 \pi)$.

Proof of Theorem 1.2. Since

$$
\mathrm{P}\left(\boldsymbol{S}_{j}(\varepsilon) \subseteq \boldsymbol{S}_{j}^{r}\right)=\mathrm{P}\left(\boldsymbol{S}_{1}(\varepsilon) \subseteq \boldsymbol{S}_{1}^{r}\right)
$$

for all $j=1, \ldots, k$, we need only prove that

$$
\lim _{r \rightarrow \infty} \mathrm{P}\left(S_{1}(\varepsilon) \subseteq S_{1}^{r}\right)=1 .
$$

To do so, for each $j=1, \ldots, k$ let $\alpha_{j}^{k}:=\pi(j-1) / k, \vec{e}_{j}^{k}:=\mathrm{e}^{2 \mathrm{i} \alpha_{j}^{k}}$, and $A_{r}:=\bigcap_{j=1}^{k} A_{r}^{j}$, where

$$
A_{r}^{j}:=\bigcap_{l \neq j}\left\{H^{\alpha_{j}^{k}}\left(r \vec{e}_{l}^{k}, r \vec{e}_{j}^{k}\right)>0\right\} .
$$

Let $\alpha_{k}^{+\varepsilon}:=\pi / k-\varepsilon$ and $\alpha_{k}^{-\varepsilon}:=(2 \pi-\pi / k)+\varepsilon$, and set

$$
B_{r}(\varepsilon):=\bigcap_{j=2, \ldots, k}\left\{H^{\alpha_{k}^{+\varepsilon}}\left(r \vec{e}_{j}^{k}, r \vec{e}_{1}^{k}\right)>0 \text { and } H^{\alpha_{k}^{-\varepsilon}}\left(r \vec{e}_{j}^{k}, r \vec{e}_{1}^{k}\right)>0\right\} .
$$

By Theorem 1.4,

$$
\lim _{r \rightarrow \infty} \mathrm{P}\left(A_{r} \cap B_{r}(\varepsilon)\right)=1 .
$$

The connectivity of the regions $\boldsymbol{B}_{j}^{r}$ yields $\boldsymbol{S}_{1}(\varepsilon) \subseteq \boldsymbol{S}_{1}^{r}$ on $A_{r} \cap B_{r}(\varepsilon)$. Together with (2.2) this yields (2.1), and the proof of Theorem 1.2 is complete. 


\section{Auxiliary results}

\subsection{The probability space}

During the subsequent proofs we will consider the following construction of $(\Omega, \mathcal{F}, P)$, the underlying probability space of our first passage percolation model. Let $\boldsymbol{u}_{0}=(0,0), \boldsymbol{u}_{2}, \ldots$ be an ordering of $\mathbb{Z}^{2}$, and for each $k \geq 1$ let

$$
\boldsymbol{B}_{k}:=\boldsymbol{u}_{k}+\left[-\frac{1}{2}, \frac{1}{2}\right]^{2},
$$

that is, a closed box of unit side length centered at $\boldsymbol{u}_{k}$. Consider

$$
\mathcal{N}=\left\{N_{k}: k \geq 1\right\},
$$

a collection of independent, identically distributed Poisson random variables with intensity 1;

$$
u_{k}=\left\{U_{k, l}: l \geq 1\right\},
$$

a collection of independent random points in the plane such that $U_{k, l}$ has a uniform distribution in the square box $\boldsymbol{B}_{k}$; and

$$
\mathcal{T}_{k}=\left\{\tau_{k, l}^{m, n}: l \geq 1, m \geq k, n \geq 1, \text { and } n>l \text { for } k=m\right\},
$$

a collection of independent, identically distributed, nonnegative random variables with common distribution $\mathbb{F}$ (the passage time distribution). We require that these collections be independent of each other.

To determine the vertex set $\mathscr{D}_{\mathrm{v}}=\mathcal{P}$, in each square box $\boldsymbol{B}_{k}$ we put $N_{k}$ points given by $U_{k, 1}, \ldots, U_{k, N_{k}}$. This procedure determines a Poisson point process $\mathcal{P}$ from the collections $\mathcal{N}$ and $U_{k}$ with $k \geq 1$. Given that $\boldsymbol{e} \in \mathscr{D}_{\mathrm{e}}$, we know that there exists a unique pair $\left(U_{k, l}, U_{m, n}\right)$, with either $m>k$ or $m=k$ and $n>l$, such that $\boldsymbol{e}=\left(U_{k, l}, U_{m, n}\right)$. Set $\tau_{\boldsymbol{e}}=\tau_{k, l}^{m, n}$.

For each $k \geq 1$, denote by $\left(\Omega^{k}, \mathcal{F}^{k}, \mathrm{P}^{k}\right)$ the probability space induced by the random variable $N_{k}$ and the collections $U_{k}$ and $\widetilde{T}_{k}$. The probability space $(\Omega, \mathcal{F}, \mathrm{P})$ is defined to be the product space of the $\left(\Omega^{k}, \mathcal{F}^{k}, \mathrm{P}^{k}\right), k \geq 1$.

An important step in the construction of the Busemann function is the proof of the coalescence behavior of semi-infinite geodesics with the same asymptotic orientation. In this proof, we will use the following modification lemma. Let $K$ be the collection of all finite sequences

$$
I=\left(\left(k_{j}, l_{j}, m_{j}, n_{j}\right)\right)_{j=1, \ldots, q} \in\left(\mathbb{N}^{4}\right)^{q},
$$

where $q \geq 1,\left(k_{j}, l_{j}, m_{j}, n_{j}\right) \neq\left(k_{i}, l_{i}, m_{i}, n_{i}\right)$ for $j \neq i, k_{1} \leq \cdots \leq k_{q}$, and either $k_{j}<$ $m_{j}$ or $l_{j}<n_{j}$. To each $I \in K$ corresponds a random vector $\left(\tau_{k_{j}, l_{j}}^{m_{j}, n_{j}}\right)_{j=1, \ldots, q}$. We denote by $\left(\Omega_{I}, \mathcal{F}_{I}, \mathrm{P}_{I}\right)$ the probability space induced by this random vector. Let

$$
\hat{\Omega}_{I}:=\left\{\hat{\omega}_{I}: \text { there exists an } \omega_{I} \in \Omega_{I} \text { with }\left(\hat{\omega}_{I}, \omega_{I}\right) \in \Omega\right\},
$$

and denote by $\hat{\mathrm{P}}_{I}$ the probability law $\mathrm{P}$ restricted to this subset. For each $I \in K, A \subseteq \Omega$, and $\omega_{1} \in \hat{\Omega}_{I}$, define

$$
A_{I, \omega_{1}}:=\left\{\omega_{2} \in \Omega_{I}: \omega=\left(\omega_{1}, \omega_{2}\right) \in A\right\} .
$$

Let $\left\{R_{I}: I \in K\right\}$ be a family of events $R_{I} \in \mathcal{F}_{I}$ such that $\mathrm{P}_{I}\left(R_{I}\right)>0$ for all $I$. Then define the map

$$
\Phi_{I}(A):=\left\{\omega_{1} \in \hat{\Omega}_{I}: \mathrm{P}_{I}\left(A_{I, \omega_{1}}\right)>0\right\} \times R_{I}
$$


on $\mathcal{F}$. Suppose that $W(\omega)$ is a random element of $K$, which may be interpreted as the set of indices (edges) whose passage time value will be modified. For $A \subseteq \Omega$, let

$$
\tilde{\Phi}(A):=\bigcup_{I \in K}\left(\left\{\omega_{1} \in \hat{\Omega}_{I}: A(I)_{I, \omega_{1}} \neq \varnothing\right\} \times R_{I}\right),
$$

where $A(I):=A \cap\{W=I\}$.

Lemma 3.1. For each $A \in \mathcal{F}, \tilde{\Phi}(A)$ contains the union $\Phi(A) \in \mathcal{F}$ defined as

$$
\Phi(A):=\bigcup_{I \in K} \Phi_{I}(A(I)) .
$$

Furthermore, if $\mathrm{P}(A)>0$ then $\mathrm{P}(\Phi(A))>0$.

Proof. If $\mathrm{P}_{I}\left(A(I)_{I, \omega_{1}}\right)>0$ then $A(I)_{I, \omega_{1}} \neq \varnothing$ and, so, $\Phi(A) \subseteq \tilde{\Phi}(A)$. Since $K$ is countable and $A=\bigcup_{I \in K} A(I)$, if $\mathrm{P}(A)>0$ then there exists an $I \in K$ such that $\mathrm{P}(A(I))>0$. For this $I$, by Fubini's theorem,

$$
0<\mathrm{P}(A(I))=\int_{\hat{\Omega}_{I}} \mathrm{P}_{I}\left(A(I)_{I, \omega_{1}}\right) \hat{\mathrm{P}}_{I}\left(\mathrm{~d} \omega_{1}\right) .
$$

Let $\hat{A}_{I}:=\left\{\omega_{1}: \mathrm{P}_{I}\left(A(I)_{I, \omega_{1}}\right)>0\right\}$. By (3.2), $\hat{\mathrm{P}}_{I}\left(\hat{A}_{I}\right)>0$. According to the definition of $\Phi_{I}$,

$$
\mathrm{P}\left(\Phi_{I}(A(I))\right)=\hat{\mathrm{P}}_{I}\left(\hat{A}_{I}\right) \mathrm{P}_{I}\left(R_{I}\right)>0 .
$$

Since $\Phi_{I}(A(I)) \subseteq \Phi(A)$, we conclude that $\mathrm{P}(\Phi(A))>0$.

\subsection{Some geometrical aspects of Delaunay triangulations}

In this subsection we study some geometrical aspects of Delaunay triangulations. Let $\boldsymbol{x}, \boldsymbol{y} \in \mathbb{R}^{2}$. We denote by $[\boldsymbol{x}, \boldsymbol{y}]$ the line segment connecting $\boldsymbol{x}$ and $\boldsymbol{y}$. We construct a path $\gamma(\boldsymbol{x}, \boldsymbol{y}):=\left(\boldsymbol{v}_{1}, \ldots, \boldsymbol{v}_{k}\right)$ in $\mathscr{D}$ connecting $\boldsymbol{v}(\boldsymbol{x})$ and $\boldsymbol{v}(\boldsymbol{y})$, as follows. Set $\boldsymbol{v}_{1}:=\boldsymbol{v}(\boldsymbol{x})$. If $\boldsymbol{v}_{1} \neq \boldsymbol{v}(\boldsymbol{y})$ then let $\boldsymbol{v}_{2}$ be the (almost surely) unique nearest neighbor of $\boldsymbol{v}_{1}$ such that the edge of $\boldsymbol{C}_{\boldsymbol{v}_{1}}$ that is perpendicular to the line segment $\left[\boldsymbol{v}_{1}, \boldsymbol{v}_{2}\right]$ crosses $[\boldsymbol{x}, \boldsymbol{y}]$. Given $\boldsymbol{v}_{l}, l \geq 1$, if $\boldsymbol{v}_{l} \neq \boldsymbol{v}(\boldsymbol{y})$ then let $\boldsymbol{v}_{l+1}$ be the (almost surely) unique nearest neighbor of $\boldsymbol{v}_{l}$, different from $\boldsymbol{v}_{l-1}$, such that the edge of $\boldsymbol{C}_{\boldsymbol{v}_{l}}$ that is perpendicular to $\left[\boldsymbol{v}_{l}, \boldsymbol{v}_{l+1}\right]$ crosses $[\boldsymbol{x}, \boldsymbol{y}]$; otherwise, we set $k:=l$ and the construction is finished. We denote by $\left|\gamma\left(\mathbf{0}, n \vec{e}_{1}\right)\right|$ the number of edges in $\gamma\left(\mathbf{0}, n \vec{e}_{1}\right)$.

For $z \in \mathbb{R}^{2}$ and $L>0$, let

$$
\boldsymbol{B}_{z}^{L}:=L z+[-L / 2, L / 2] .
$$

For $n>0$, consider the set $\varepsilon_{n}$ composed of edges $(\boldsymbol{v}, \overline{\boldsymbol{v}}) \in \mathcal{D}_{\mathrm{e}}$ with $\boldsymbol{C}_{\boldsymbol{v}} \cap \boldsymbol{B}_{z}^{1} \neq \varnothing$ or $\boldsymbol{C}_{\overline{\boldsymbol{v}}} \cap \boldsymbol{B}_{z}^{1} \neq \varnothing$ for some $z \in\left[\mathbf{0}, n \vec{e}_{1}\right]$. We denote by $\left|\mathcal{E}_{n}\right|$ the number of edges in $\mathcal{E}_{n}$.

Lemma 3.2. There exist constants $z_{j}, c_{j}>0, j=1,2$, such that, for all $n \geq 1$,

$$
\mathrm{P}\left(\left|\gamma\left(\mathbf{0}, n \vec{e}_{1}\right)\right| \geq z n\right) \leq \mathrm{e}^{-c_{1} z n} \text { for } z \geq z_{1}
$$

and

$$
\mathrm{P}\left(\left|\mathcal{E}_{n}\right| \geq z n\right) \leq \mathrm{e}^{-c_{2} z n} \text { for } z \geq z_{2}
$$




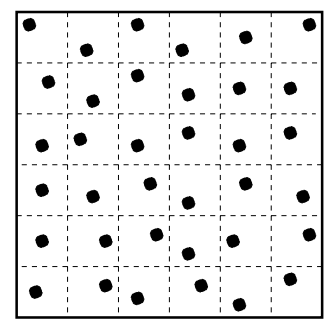

FIGURE 3: Renormalization: a full box.

The proof of this lemma is performed using renormalization ideas developed in [17]. To avoid unnecessary repetition, we give a sketch of the proof and leave the details, which can be filled in by following the arguments in the proof of Proposition 2.2 of [17] (which is exactly the proof of (3.3)), to the reader.

Proof of Lemma 3.2. For $z \in \mathbb{Z}^{2}$ and $L>0$, divide a square box $\boldsymbol{B}_{z}^{L}$ into thirty-six subboxes, say $\boldsymbol{B}_{1}, \ldots, \boldsymbol{B}_{36}$, of the same side length. We describe $\boldsymbol{B}$ as a full box if all thirty-six sub-boxes contain at least one Poisson point (see Figure 3 ).

We say that $\Lambda:=\left(\boldsymbol{B}_{z_{1}}^{L}, \ldots, \boldsymbol{B}_{z_{k}}^{L}\right)$ is a circuit of boxes if $\left(z_{1}, \ldots, z_{k}\right)$ is a circuit in $\mathbb{Z}^{2}$ (in the usual sense). Let $\lambda$ be the closed polygonal path composed of the line segments connecting $L z_{j}$ and $L z_{j+1}, j=1, \ldots, k-1$, together with $\left[z_{k}, z_{1}\right]$. To each circuit $\Lambda$ we associate two subsets of the plane: $\Lambda^{\text {in }}$ denotes the interior of the bounded component of $\mathbb{R}^{2} \backslash \bigcup_{j=1}^{k} \boldsymbol{B}_{z_{j}}^{L}$, while $\lambda^{\text {in }}$ denotes the interior of the bounded component of $\mathbb{R}^{2} \backslash \lambda$. Now, assume that $\Lambda:=\left(\boldsymbol{B}_{z_{j}}^{L}\right)_{j=1}^{k}$ is a circuit composed of full boxes. From Lemma 2.1 of [17], we have the following geometrical property: if $\boldsymbol{C}_{\boldsymbol{v}} \cap \Lambda^{\text {in }} \neq \varnothing$ then $\boldsymbol{C}_{\boldsymbol{v}} \subseteq \lambda^{\text {in }}$. One important consequence of this is that the set of vertices connected by $\gamma\left(\mathbf{0}, n \vec{e}_{1}\right)$ and the set of vertices used by $\varepsilon_{n}$ are both contained in the region, $\boldsymbol{R}_{n}$, delineated by the smallest circuit of full boxes surrounding the line segment $\left[\mathbf{0}, n \vec{e}_{1}\right]$. Therefore, to prove Lemma 3.2 it is enough to prove the analogous decay of the number of Poisson points in $\boldsymbol{R}_{n}$. (Recall that, according to the Euler formula, the number of edges and vertices in a triangulation are of the same order.)

Notice that, since each box is full independently of the others and the probability that a box is full goes to 1 as $L$ goes to $\infty$, for a fixed, large $L_{0}>0$ the probability that $\boldsymbol{R}_{n}$ contains more than $z n$ boxes decays as $\mathrm{e}^{-c z n}$.

Now, the number of points in $\boldsymbol{R}_{n}$, say $R_{n}$, is the sum of independent Poisson random variables and is less than or equal to $M_{m}$, the maximum of the number of points in $\boldsymbol{R}$ over all connected regions $\boldsymbol{R}$ intersecting at most $m$ boxes and containing the origin, $\mathbf{0}$. Thus, on the event that $\boldsymbol{R}_{n}$ contains fewer than $z n$ boxes, we have $R_{n} \leq M_{z n}$. On the other hand, $M_{m}$ can be viewed as a greedy lattice animal model, and for such a model we can also show that, for large $\bar{c}>0$, the probability that $M_{m} \geq \bar{c} m$ decays as $\mathrm{e}^{-c m}$ (see Lemma 2.3 of [17]).

By combining the arguments in the two last paragraphs we find that the probability that the number of points in $\boldsymbol{R}_{n}$ is greater than $z n$ also decays as $\mathrm{e}^{-c z n}$, for some constant $c>0$ and sufficiently large $z$.

Let $T_{\mathscr{D}}$ denote the graph metric on $\mathscr{D}$, i.e. for $\boldsymbol{v}, \overline{\boldsymbol{v}} \in \mathscr{D}_{\mathrm{v}}, T_{\mathscr{D}}(\boldsymbol{v}, \overline{\boldsymbol{v}})$ is the minimum number of edges that one path can cross in going from $\boldsymbol{v}$ to $\overline{\boldsymbol{v}}$. Notice that $T_{\mathscr{D}}(\boldsymbol{v}, \overline{\boldsymbol{v}})$ is the first passage time between $\boldsymbol{v}$ and $\overline{\boldsymbol{v}}$ if we associate to each edge $\boldsymbol{e}$ the passage time value 1 . For each $\boldsymbol{A}, \boldsymbol{B} \subseteq \mathbb{R}^{2}$, we set $T_{\mathscr{D}}(\boldsymbol{A}, \boldsymbol{B})$ to be the minimum of $T_{\mathscr{D}}(\boldsymbol{v}, \tilde{\boldsymbol{v}})$ over all pairs $\boldsymbol{v}$ and $\tilde{\boldsymbol{v}}$ such that $\boldsymbol{C}_{\boldsymbol{v}} \cap \boldsymbol{A} \neq \varnothing$ and $\boldsymbol{C}_{\tilde{\boldsymbol{v}}} \cap \boldsymbol{B} \neq \varnothing$. From the shape theorem, we have the following result. 
Lemma 3.3. There exists a $v \in(0, \infty)$ such that, almost surely,

$$
\lim _{n \rightarrow \infty} \frac{T_{\mathscr{D}}\left(\boldsymbol{A}, n \mathrm{e}^{\mathrm{i} \alpha}+\boldsymbol{B}\right)}{n}=v .
$$

We note that $v$ does not depend either on $\boldsymbol{A}$ and $\boldsymbol{B}$ or on $\alpha \in[0,2 \pi)$. We also see that, with $\lambda \equiv \lambda(\mathbb{F})$ denoting the supremum of the support of $\mathbb{F}$, we have

$$
\mu(\mathbb{F}) \leq \mathrm{E}\left(\tau_{\mathrm{e}}\right) v<\lambda \nu
$$

(we must assume that $\mathbb{F}$ is not concentrated at one point, which is the case here since $\mathbb{F}$ is continuous).

We shall also use the following lemma, which is Equation (5.2) of Lemma 5.2 of [12].

Lemma 3.4. For $\xi \in(0,1)$ and $r>0$, let $A_{\xi, r}$ be the event that there exists an $x \in \mathbb{R}^{2}$ with $|\boldsymbol{x}| \leq 2 r$ and $|\boldsymbol{x}-\boldsymbol{v}(\boldsymbol{x})| \geq r^{\xi}$. Then, for some constant $c_{1}>0$,

$$
\mathrm{P}\left(A_{\xi, r}\right) \leq c_{1} \exp \left(-r^{2 \xi}\right) .
$$

\section{3. $\delta$-straightness of semi-infinite paths}

Recall that for $\alpha \in[0,2 \pi)$ we have defined a self-avoiding path $\left(\boldsymbol{x}_{n}\right)_{n \geq 1}$, with vertices in $\mathbb{R}^{2}$ and such that $\left|\boldsymbol{x}_{n}\right| \rightarrow \infty$ as $n \rightarrow \infty$, to be an $\alpha$-path if

$$
\lim _{n \rightarrow \infty} \frac{\boldsymbol{x}_{n}}{\left|\boldsymbol{x}_{n}\right|}=\mathrm{e}^{\mathrm{i} \alpha}:=(\cos \alpha, \sin \alpha),
$$

and that a sufficient condition for a path $\left(\boldsymbol{x}_{n}\right)_{n \geq 1}$ to be an $\alpha$-path for some $\alpha \in[0,2 \pi)$ is that, for some fixed $\delta \in(0,1)$ and $c>0$, and for large enough $n$,

$$
\operatorname{ang}\left(\boldsymbol{x}_{n}, \boldsymbol{x}_{m}\right) \leq\left|\boldsymbol{x}_{n}\right|^{-\delta} \text { for } m>n
$$

( $\delta$-straightness). A sufficient condition for $\delta$-straightness is given by the next lemma, which is exactly Lemma 2.7 of [12]. We denote by $d(\boldsymbol{x}, A)$ the Euclidean distance between $\boldsymbol{x}$ and $A \subseteq \mathbb{R}^{2}$.

Lemma 3.5. If $\left(\boldsymbol{x}_{n}\right)_{n \geq 1}$ is a sequence of points in $\mathbb{R}^{2}$ such that $\left|\boldsymbol{x}_{n}\right| \rightarrow \infty$ as $n \rightarrow \infty$ and such that, for all large $n$,

$$
\left|\boldsymbol{x}_{n+1}-\boldsymbol{x}_{n}\right| \leq\left|\boldsymbol{x}_{n}\right|^{1-\delta} \text { and } d\left(\boldsymbol{x}_{n},\left[\boldsymbol{x}_{1}, \boldsymbol{x}_{m}\right]\right) \leq\left|\boldsymbol{x}_{m}\right|^{1-\delta} \quad \text { for } m>n,
$$

then there exists a constant $c>0$ such that, for all sufficiently large $n$,

$$
\operatorname{ang}\left(\boldsymbol{x}_{n}, \boldsymbol{x}_{m}\right) \leq c\left|\boldsymbol{x}_{n}\right|^{-\delta} \text { for } m>n \text {. }
$$

We also consider the $\delta$-straightness property for trees (we have the tree of infection in mind), as follows. For $\varepsilon \in[0, \pi)$, let

$$
\boldsymbol{C}(\boldsymbol{x}, \varepsilon):=\left\{\boldsymbol{y} \in \mathbb{R}^{2} \backslash\{0\}: \operatorname{ang}(\boldsymbol{y}, \boldsymbol{x}) \leq \varepsilon\right\} .
$$

If $\mathcal{T}$ is a tree embedded in $\mathbb{R}^{2}$ then, for each pair $\boldsymbol{v}, \tilde{\boldsymbol{v}} \in \mathcal{T}$, let $\mathcal{R}_{\text {out }}(\boldsymbol{v}, \tilde{\boldsymbol{v}})$ be the set of all $\hat{\boldsymbol{v}} \in \mathcal{T}$ such that the unique path in $\mathcal{T}$ connecting $\boldsymbol{v}$ and $\hat{\boldsymbol{v}}$ touches $\tilde{\boldsymbol{v}}$. For $\delta \in(0,1)$, we define $\mathcal{T}$ to be $\delta$-straight at $\boldsymbol{v}$ if, for all but finitely many $\tilde{\boldsymbol{v}} \in \mathcal{T}$,

$$
\mathcal{R}_{\text {out }}(\boldsymbol{v}, \tilde{\boldsymbol{v}}) \subseteq \boldsymbol{v}+C\left(\tilde{\boldsymbol{v}}-\boldsymbol{v}, c|\tilde{\boldsymbol{v}}-\boldsymbol{v}|^{-\delta}\right) .
$$

We say that a subset $\mathcal{P}$ of $\mathbb{R}^{2}$ is omnidirectional if, for all $M>0$, the set composed of unit vectors $\boldsymbol{v} /|\boldsymbol{v}|$ with $\boldsymbol{v} \in \mathcal{P}$ and $|\boldsymbol{v}|>M$ is dense in $\boldsymbol{S}^{1}$. The following lemma, which is Proposition 2.8 of [12], states that $\delta$-straightness implies the existence of an asymptotic orientation. 
Lemma 3.6. Assume that $\mathcal{T}$ is a tree embedded in $\mathbb{R}^{2}$ whose vertex set is locally finite but omnidirectional and such that every vertex has finite degree. Assume further that, for some vertex $\boldsymbol{v}, \mathcal{T}$ is $\delta$-straight at $\boldsymbol{v}$. Then the following statements hold.

1. Every semi-infinite path in $\mathcal{T}$ starting at $\boldsymbol{v}$ has an asymptotic orientation.

2. For every $\alpha \in[0,2 \pi)$, there exists at least one semi-infinite path in $\mathcal{T}$ that starts at $\boldsymbol{v}$ and has asymptotic orientation $\mathrm{e}^{\mathrm{i} \alpha}$.

3. Every semi-infinite path $\left(\boldsymbol{v}_{n}\right)_{n \geq 1}$ in $\mathcal{T}$ starting at $\boldsymbol{v}$ is $\delta$-straight about its asymptotic orientation, $\mathrm{e}^{\mathrm{i} \alpha}$, i.e. $\operatorname{ang}\left(\boldsymbol{v}_{n}, \mathrm{e}^{\mathrm{i} \alpha}\right)<c\left|\boldsymbol{v}_{n}\right|^{-\delta}$ eventually.

\section{Semi-infinite geodesics and the Busemann function}

\subsection{Semi-infinite geodesics: existence}

Recall that a path $\rho=\left(\boldsymbol{v}_{1}, \boldsymbol{v}_{2}, \ldots\right)$ in $\mathscr{D}$ is a semi-infinite geodesic if, for all $\boldsymbol{v}_{j}, \boldsymbol{v}_{k} \in \rho$, the path $\left(\boldsymbol{v}_{j}, \boldsymbol{v}_{j+1}, \ldots, \boldsymbol{v}_{k}\right)$ is the unique geodesic connecting $\boldsymbol{v}_{j}$ and $\boldsymbol{v}_{k}$. Semi-infinite geodesics that start at $v \in \mathscr{D}_{\mathrm{v}}$ and have asymptotic orientation $\mathrm{e}^{\mathrm{i} \alpha}$ are denoted by $\rho_{\boldsymbol{v}}(\alpha)$.

Proposition 4.1. Let $\Omega_{2}$ be the event that for all semi-infinite geodesics $\rho$ there exists an $\alpha \equiv \alpha(\rho) \in[0,2 \pi)$ such that $\rho$ is an $\alpha$-path, and that for all $\alpha \in[0,2 \pi)$ and for all $v \in \mathscr{D}_{\mathrm{v}}$ there exists at least one geodesic that starts at $\boldsymbol{v}$ and has asymptotic orientation $\mathrm{e}^{\mathrm{i} \alpha}$. Under (1.1), $\mathrm{P}\left(\Omega_{2}\right)=1$.

The first step in showing the existence of semi-infinite geodesics is the following result on the fluctuations of $T$, which is exactly Corollary 1.1 of [17].

Lemma 4.1. Under (1.1), for all $\kappa \in\left(\frac{1}{2}, 1\right)$ there exist constants $\delta, c_{j}>0$ such that, for all $r \geq 1$ and $s \in\left[c_{1}(\log r)^{1 / \delta}, c_{2} r^{\kappa}\right]$,

$$
\mathrm{P}\left(\left|T\left(\mathbf{0}, r \vec{e}_{1}\right)-\mu r\right| \geq s r^{\kappa}\right) \leq \exp \left(-c_{3} s^{\delta}\right) .
$$

The second step is to parallel the analysis in [15] to prove that control of the fluctuations of $T$ can give control of the fluctuations about the line segment $\left[\mathbf{0}, r \vec{e}_{1}\right]$ of a minimizing path connecting $\mathbf{0}$ and $r \vec{e}_{1}$. More precisely, for $\xi \in(0,1)$ let

$$
C_{r}^{\xi}:=\left\{\boldsymbol{x} \in \mathbb{R}^{2}: d\left(\boldsymbol{x},\left[\mathbf{0}, r \vec{e}_{1}\right]\right) \leq r^{\xi}\right\} .
$$

Lemma 4.2. For all $\xi \in\left(\frac{3}{4}, 1\right)$, there exist constants $c, \delta>0$ such that, for all $r \geq 1$,

$$
\mathrm{P}\left(\rho\left(\mathbf{0}, r \vec{e}_{1}\right) \nsubseteq \boldsymbol{C}_{r}^{\xi}\right) \leq \exp \left(-c r^{\delta}\right) .
$$

Proof. Let $\kappa \in\left(\frac{1}{2}, 1\right)$ and $\tilde{\kappa} \in(\kappa, 1)$ and set $\xi=(\tilde{\kappa}+1) / 2$. Let

$$
\boldsymbol{C}_{r}^{1, \xi}:=\left\{\boldsymbol{x} \in \mathbb{R}^{2} \backslash \boldsymbol{C}_{r}^{\xi}: d\left(\boldsymbol{x}, \boldsymbol{C}_{r}^{\xi}\right)<r^{\xi}\right\} .
$$

Denote by $F_{r}$ the event defined by the following properties:

- $\boldsymbol{v}_{\mathbf{0}}, \boldsymbol{v}_{r \vec{e}_{1}} \in \boldsymbol{C}_{r}^{\xi}$ and

- for all edges $\boldsymbol{e}=(\boldsymbol{v}, \tilde{\boldsymbol{v}})$ with $|\boldsymbol{v}| \leq 2 r$ or $|\tilde{\boldsymbol{v}}| \leq 2 r$, we have $|\boldsymbol{v}-\tilde{\boldsymbol{v}}| \leq r^{\xi}$. 
Note that $F_{r}^{\mathrm{c}} \subseteq A_{\xi, r / 3}$ (the latter as defined in Lemma 3.4) and, thus,

$$
\mathrm{P}\left(F_{r}^{\mathrm{c}}\right) \leq c_{1} \exp \left(-\left(\frac{r}{3}\right)^{2 \xi}\right) .
$$

For each $z \in \mathbb{Z}^{2}$, consider the random variable

$$
T_{z}:=\max _{|\boldsymbol{v}-z| \leq 1}\{T(z, v)\}
$$

We claim that, under (1.1), for some constants $c_{2}, c_{3}>0$,

$$
\mathrm{P}\left(T_{z} \geq r^{\kappa}\right)=\mathrm{P}\left(T_{0} \geq r^{\kappa}\right) \leq c_{2} \exp \left(-c_{3} r^{\kappa}\right) .
$$

To see this, notice that $T_{\mathbf{0}} \leq \sum_{\boldsymbol{e} \in \mathcal{E}_{1}} \tau_{\boldsymbol{e}}$, where $\mathcal{E}_{1}$ is the set of edges $\boldsymbol{e}=(\boldsymbol{v}, \overline{\boldsymbol{v}})$ in $\mathscr{D}_{\mathrm{e}}$ with $\boldsymbol{C}_{\boldsymbol{v}} \cap \boldsymbol{B}_{\mathbf{0}}^{1} \neq \varnothing$ or $\boldsymbol{C}_{\overline{\boldsymbol{v}}} \cap \boldsymbol{B}_{\mathbf{0}}^{1} \neq \varnothing$. By Lemma 3.2, E(exp $\left.\left(a\left|\varepsilon_{1}\right|\right)\right)<\infty$ for some $a>0$. Combining this with assumption (1.1) and the independence between the Poisson point process and the passage time distribution, we obtain (4.2).

Now,

$$
\begin{aligned}
& \left\{\rho\left(\mathbf{0}, r \vec{e}_{1}\right) \nsubseteq \boldsymbol{C}_{r}^{\xi}\right\} \cap F_{r} \\
& \quad \subseteq\left\{\text { there exists a } \boldsymbol{v} \in \mathscr{D}_{\mathrm{v}} \cap \boldsymbol{C}_{r}^{1, \xi} \text { such that } T(\mathbf{0}, \boldsymbol{v})+T\left(\boldsymbol{v}, r \vec{e}_{1}\right)=T\left(\mathbf{0}, r \vec{e}_{1}\right)\right\} \\
& \quad \subseteq A_{\xi}(r),
\end{aligned}
$$

where

$$
A_{\xi}(r):=\left\{\text { there exists a } z \in \mathbb{Z}^{2} \cap \boldsymbol{C}_{r}^{1, \xi} \text { such that } T(\mathbf{0}, z)+T\left(z, r \vec{e}_{1}\right) \leq T\left(\mathbf{0}, r \vec{e}_{1}\right)+2 T_{z}\right\} .
$$

Let

$$
\Delta\left(z, r \vec{e}_{1}\right):=\mu\left|z-r \vec{e}_{1}\right|+\mu|z|-\mu\left|r \vec{e}_{1}\right|
$$

Then

$$
T(\mathbf{0}, z)+T\left(z, r \vec{e}_{1}\right) \leq T\left(\mathbf{0}, r \vec{e}_{1}\right)+2 T_{z}
$$

if and only if

$$
\Delta\left(z, r \vec{e}_{1}\right) \leq T\left(\mathbf{0}, r \vec{e}_{1}\right)-\mu r+\mu|z|-T(\mathbf{0}, z)+\mu\left|z-r \vec{e}_{1}\right|-T\left(z, r \vec{e}_{1}\right)+2 T_{z} .
$$

This implies that $A_{\xi}(r) \subseteq \bigcup_{j=0}^{3} A_{j}(r)$, where

$$
\begin{aligned}
& A_{0}(r):=\left\{\text { there exists a } z \in \mathbb{Z}^{2} \cap C_{r}^{1, \xi} \text { such that } T_{z} \geq \Delta\left(z, r \vec{e}_{1}\right) / 8\right\}, \\
& A_{1}(r):=\left\{\text { there exists a } z \in \mathbb{Z}^{2} \cap C_{r}^{1, \xi} \text { such that }\left|T\left(z, r \vec{e}_{1}\right)-\mu\right| z-r \vec{e}_{1}|| \geq \Delta\left(z, r \vec{e}_{1}\right) / 4\right\}, \\
& A_{2}(r):=\left\{\text { there exists a } z \in \mathbb{Z}^{2} \cap C_{r}^{1, \xi} \text { such that }|T(\mathbf{0}, z)-\mu| z|| \geq \Delta\left(z, r \vec{e}_{1}\right) / 4\right\}, \\
& A_{3}(r):=\left\{\text { there exists a } z \in \mathbb{Z}^{2} \cap C_{r}^{1, \xi} \text { such that }\left|T\left(\mathbf{0}, r \vec{e}_{1}\right)-\mu\right| r \vec{e}_{1}|| \geq \Delta\left(z, r \vec{e}_{1}\right) / 4\right\} .
\end{aligned}
$$

Combining this with (4.3), we find that

$$
\mathrm{P}\left(\rho\left(\boldsymbol{0}, r \vec{e}_{1}\right) \nsubseteq \boldsymbol{C}_{r}^{\xi}\right) \leq \mathrm{P}\left(F_{r}^{\mathrm{c}}\right)+\sum_{j=0}^{3} \mathrm{P}\left(A_{j}(r)\right) .
$$


Note that there exist constants $b_{1}, b_{2}>0$ such that, for sufficiently large $r>0$ and $z \in \mathbb{Z}^{2} \cap \boldsymbol{C}_{r}^{1, \xi}$, we have

$$
b_{1} r^{\tilde{\kappa}}=b_{1} r^{2 \xi-1} \leq \Delta\left(z, r \vec{e}_{1}\right) \leq b_{2} r^{\xi}=b_{2} r^{(\tilde{\kappa}+1) / 2}
$$

and

$$
r^{\xi} \leq|z|, \quad\left|z-r \vec{e}_{1}\right| \leq 2 r .
$$

Together with Lemma (4.1), for some constant $c_{1}>0$, (4.5) and (4.6) yield

$$
\mathrm{P}\left(A_{j}(r)\right) \leq \exp \left(-c_{1} r^{\delta}\right)
$$

Combining (4.4) with (4.1), (4.2), and (4.7) completes the proof of the lemma.

For $\boldsymbol{v} \in \mathscr{D}_{\mathrm{v}}$, let $\mathcal{T}_{\boldsymbol{v}}$ be the union over all $\tilde{\boldsymbol{v}} \in \mathscr{D}_{\mathrm{v}}$ of the unique geodesic between $\boldsymbol{v}$ and $\tilde{\boldsymbol{v}}$ (the tree of infection at $\boldsymbol{v}$ ). Then $\mathcal{T}_{\boldsymbol{v}}$ is a tree spanning all $\mathscr{D}_{\mathrm{v}}$. Thus, the third step in showing the existence and convergence of semi-infinite geodesics is to use Lemma 4.2 and the concept of $\delta$-straightness for trees discussed above.

Proof of Proposition 4.1. Combining Lemma 4.2 and Lemma 3.4 with the Borel-Cantelli lemma, we find that, for all $\delta=1-\xi \in\left(0, \frac{1}{4}\right)$, the assumptions of Lemma 3.5 almost surely hold for all semi-infinite paths (geodesics) $\left(\boldsymbol{v}_{n}\right)_{n \geq 1}$ in $\mathcal{T}_{\boldsymbol{v}}$. Thus, $\mathcal{T}_{\boldsymbol{v}}$ is $\delta$-straight at $\boldsymbol{v}$. Since, with probability 1 , a realization of the Poisson point process is omnidirectional, together with Lemma 3.6 this yields Proposition 4.1.

Remark 4.1. Let $\xi \in\left(\frac{3}{4}, 1\right)$. The almost-sure $(1-\xi)$-straightness of the tree of infection also implies that, for all $\alpha \in[0,2 \pi)$, if $\left(\boldsymbol{v}_{1}, \boldsymbol{v}_{2}, \ldots\right)$ is a semi-infinite geodesic with asymptotic orientation $\mathrm{e}^{\mathrm{i} \alpha}$, then

$$
\operatorname{ang}\left(\boldsymbol{v}_{n}, \mathrm{e}^{\mathrm{i} \alpha}\right) \leq c\left|\boldsymbol{v}_{n}\right|^{\xi-1}
$$

for sufficiently large $n$.

\subsection{Semi-infinite geodesics: uniqueness and coalescence}

Concerning uniqueness of semi-infinite geodesics, we have the following result.

Proposition 4.2. For $\alpha \in[0,2 \pi)$, let $\Omega_{3}(\alpha)$ be the event that for all $v \in \mathscr{D}_{\mathrm{v}}$ there exists at most one geodesic that starts at $\boldsymbol{v}$ and has asymptotic orientation $\mathrm{e}^{\mathrm{i} \alpha}$. Assuming only that $\mathbb{F}$ is continuous, we then have $\mathrm{P}\left(\Omega_{3}(\alpha)\right)=1$.

Proof. For $(k, l) \in \mathbb{N}^{2}$, let $A_{\alpha}(k, l)$ be the event that both $U_{k, l} \in \mathscr{D}_{\mathrm{v}}$ (or, equivalently, $N_{k} \geq l$ ) and there exist two semi-infinite geodesics that start at $\boldsymbol{v}=U_{k, l}$, have common asymptotic orientation $\mathrm{e}^{\mathrm{i} \alpha}$, and, apart from at $\boldsymbol{v}$, do not intersect each other. Then

$$
\Omega_{3}(\alpha)^{\mathrm{c}} \subseteq \bigcup_{(k, l) \in \mathbb{N}^{2}} A_{\alpha}(k, l) .
$$

Now, semi-infinite geodesics starting at the same vertex are not allowed to cross each other, and if a semi-infinite geodesic is confined between two others with common asymptotic orientation $\mathrm{e}^{\mathrm{i} \alpha}$, then it must also have asymptotic orientation $\mathrm{e}^{\mathrm{i} \alpha}$ (by planarity). Therefore, denoting by $d_{v}$ the degree of the vertex $v=U_{k, l}$, we have

$$
\left|\left\{\alpha \in[0,2 \pi): 1_{A_{\alpha}(k, l)}(\omega)=1\right\}\right| \leq d_{v}(\omega)
$$


(where $|A|$ is the cardinality of the set $A$ and $1_{\{\cdot\}}(\cdot)$ is the indicator function). In particular,

$$
\int_{[0,2 \pi)} 1_{A_{\alpha}(k, l)} \mathrm{d} \alpha=0 \quad \text { almost surely }
$$

and, so, by Fubini's theorem,

$$
\begin{aligned}
0 & \leq \int_{[0,2 \pi)} \mathrm{P}\left(\Omega_{3}(\alpha)^{\mathrm{c}}\right) \mathrm{d} \alpha=\int_{\Omega}\left(\int_{[0,2 \pi)} 1_{\Omega_{3}(\alpha)^{\mathrm{c}}} \mathrm{d} \alpha\right) \mathrm{dP} \\
& \leq \int_{\Omega}\left(\int_{[0,2 \pi)} \sum_{(k, l) \in \mathbb{N}^{2}} 1_{A_{\alpha}(k, l)} \mathrm{d} \alpha\right) \mathrm{dP}=\int_{\Omega}\left(\sum_{(k, l) \in \mathbb{N}^{2}} \int_{[0,2 \pi)} 1_{A_{\alpha}(k, l)} \mathrm{d} \alpha\right) \mathrm{dP} \\
& =0 .
\end{aligned}
$$

Consequently, there exists an $I \subseteq[0,2 \pi)$ that has total Lebesgue measure and is such that, for all $\alpha \in I, \mathrm{P}\left(\Omega_{3}(\alpha)\right)=1$. Since $\mathrm{P}\left(\Omega_{3}(\alpha)\right)$ does not depend on $\alpha$, this yields Proposition 4.2.

The last result we require to construct the Busemann function is the coalescence behavior of semi-infinite geodesics with the same asymptotic orientation.

Proposition 4.3. For $\alpha \in[0,2 \pi)$, let $\Omega_{4}(\alpha) \subseteq \Omega_{3}(\alpha)$ be the event that, for all $\boldsymbol{v}, \overline{\boldsymbol{v}} \in \mathcal{D}_{\mathrm{v}}$, if $\rho_{v}(\alpha)$ and $\rho_{\bar{v}}(\alpha)$ exist (and are unique), then they must coalesce, i.e. there exists a $\boldsymbol{c} \equiv \boldsymbol{c}(\boldsymbol{v}, \overline{\boldsymbol{v}}, \alpha) \in \mathcal{D}_{\mathrm{v}}$ such that

$$
\rho_{\boldsymbol{v}}(\alpha)=\rho(\boldsymbol{v}, \boldsymbol{c}) \cup \rho_{\boldsymbol{c}}(\alpha) \quad \text { and } \quad \rho_{\overline{\boldsymbol{v}}}(\alpha)=\rho(\overline{\boldsymbol{v}}, \boldsymbol{c}) \cup \rho_{\boldsymbol{c}}(\alpha) .
$$

Assuming only that $\mathbb{F}$ is continuous, we then have $\mathrm{P}\left(\Omega_{4}(\alpha)\right)=1$.

Note that the almost-sure statement in Proposition 4.3 holds for fixed $\alpha \in[0,2 \pi)$. As we will see later, there almost surely exists a random direction $\theta$ such that neither uniqueness nor coalescence hold. Indeed, we will show (in Subsection 4.4) that every branch of the competition interface follows one of the random directions for which coalescence does not hold. (For more on the noncoalescence of semi-infinite geodesics, see Section 1.3 of [12].)

Let $\delta(\alpha)$ denote the union over all $v \in D_{\mathrm{v}}$ of $\rho_{v}(\alpha)$. Then $\delta(\alpha)$ is a forest with, say, $N(\alpha)$ disjoint trees. Notice that on $\{N(\alpha) \leq 1\} \cap \Omega_{3}(\alpha)$ there are no site-disjoint semiinfinite geodesics with asymptotic orientations $\mathrm{e}^{\mathrm{i} \alpha}$. Proposition 4.3 will thus follow if we prove that $\mathrm{P}(N(\alpha) \leq 1)=1$. As noted by Licea and Newman [13], in this setup we can apply the Burton-Keane [1] method. This method requires several steps which we will be organized as independent assertions. To state the first, let $\delta \in \mathbb{Q}$ (the latter being the set of rational numbers) and for $i=1, \ldots, j$ let $\boldsymbol{x}_{i}=\left(x_{i}(1), x_{i}(2)\right), \tilde{\boldsymbol{x}}_{i}=\left(\tilde{x}_{i}(1), \tilde{x}_{i}(2)\right) \in \mathbb{Q}^{2}$ with $x_{1}(2) \leq \cdots \leq x_{j}(2)$ and $\tilde{x}_{1}(2) \leq \cdots \leq \tilde{x}_{j}(2)$. Assume further that $x_{i}(1) \leq-\delta$ and $\tilde{x}_{i}(1) \geq \delta$. Let $D_{\delta}(\boldsymbol{x})$ denote the Euclidean ball of radius $\delta$ centered at $\boldsymbol{x}$, and denote by $A_{\delta}\left(\boldsymbol{x}_{1}, \ldots, \boldsymbol{x}_{j}, \tilde{\boldsymbol{x}}_{1}, \ldots, \tilde{\boldsymbol{x}}_{j}\right)$ the event defined by the following properties:

- at each $D_{\delta}\left(\boldsymbol{x}_{i}\right)$ and $D_{\delta}\left(\tilde{\boldsymbol{x}}_{i}\right)$ there is a unique vertex $\boldsymbol{v}_{i}$ and, respectively, $\tilde{\boldsymbol{v}}_{i}$;

- each $\boldsymbol{e}_{i}=\left(\boldsymbol{v}_{i}, \tilde{\boldsymbol{v}}_{i}\right)$ is an edge in $\mathscr{D}_{\mathrm{e}}$, and $\boldsymbol{e}_{i} \in \rho_{\boldsymbol{v}_{i}}(0)$;

- apart from $\boldsymbol{v}_{i}, \rho_{\boldsymbol{v}_{i}}(0)$ only has vertices with strictly positive coordinates; and

- the $\rho_{\boldsymbol{v}_{i}}(0)$ are disjoint. 
Assertion 4.1. If $\mathrm{P}(N(0) \geq 2)>0$ then

$$
\mathrm{P}\left(A_{\delta}\left(\boldsymbol{x}_{1}, \boldsymbol{x}_{2}, \boldsymbol{x}_{3}, \tilde{\boldsymbol{x}}_{1}, \tilde{\boldsymbol{x}}_{2}, \tilde{\boldsymbol{x}}_{3}\right)\right)>0
$$

for some $\delta \in \mathbb{Q}$ and $\boldsymbol{x}_{i}, \tilde{\boldsymbol{x}}_{i} \in \mathbb{Q}^{2}, i=1,2,3$.

Proof. Since $\mathbb{Q}$ is enumerable, if $0<\mathrm{P}(N(0) \geq 2)$ then there exist $\delta \in \mathbb{Q}$ and $\boldsymbol{x}_{1}, \boldsymbol{x}_{2}$, $\tilde{\boldsymbol{x}}_{1}, \tilde{\boldsymbol{x}}_{2} \in \mathbb{Q}^{2}$ such that

$$
0<\mathrm{P}\left(A_{\delta}\left(\boldsymbol{x}_{1}, \boldsymbol{x}_{2}, \tilde{\boldsymbol{x}}_{1}, \tilde{\boldsymbol{x}}_{2}\right)\right) .
$$

Let $c_{\mathrm{n}}$ be the maximum of the second coordinates of $\boldsymbol{x}_{2}$ and $\tilde{\boldsymbol{x}}_{2}$ and let $c_{\mathrm{s}}$ be the minimum of the second coordinates of $\boldsymbol{x}_{1}$ and $\tilde{\boldsymbol{x}}_{1}$. Consider the rectangle

$$
\boldsymbol{R}_{0}:=[-\delta, \delta] \times\left(c_{\mathrm{s}}-\delta, c_{\mathrm{n}}+\delta\right) .
$$

Let $z_{0}$ be the circumcenter of the rectangle $\boldsymbol{R}_{0}$ and let $M_{0}$ be the vertical length of $\boldsymbol{R}_{0}$. For each $l \in \mathbb{Z}$, let $z_{l}:=z_{0}+l M_{0}(0,1), \boldsymbol{R}_{l}:=z_{l}+\boldsymbol{R}_{0}$, and

$$
A^{l}:=A_{\delta}\left(\boldsymbol{x}_{1}^{l}, \boldsymbol{x}_{2}^{l}, \tilde{\boldsymbol{x}}_{1}^{l}, \tilde{\boldsymbol{x}}_{2}^{l}\right),
$$

where $\boldsymbol{x}_{j}^{l}:=\boldsymbol{x}_{j}+z_{l} \in \boldsymbol{R}_{l}$ and $\tilde{\boldsymbol{x}}_{j}^{l}:=\tilde{\boldsymbol{x}}_{j}+z_{l}$. Thus, $\mathrm{P}\left(A^{l}\right)=\mathrm{P}\left(A^{0}\right)$. By Fatou's lemma,

$$
0<\mathrm{P}\left(A^{0}\right) \leq \mathrm{P}\left(\lim \sup _{l} A^{l}\right) \leq \mathrm{P}\left(\bigcup_{l_{1} \neq l_{2}} A^{l_{1}} \cap A^{l_{2}}\right) .
$$

Therefore, there exist $l_{1}$ and $l_{2}$ such that $0<\mathrm{P}\left(A^{l_{1}} \cap A^{l_{2}}\right)$.

Without lost of generality, assume that $l_{1}<l_{2}$. We claim that, in this case, the geodesic starting at $\boldsymbol{v}_{1}^{l_{1}}$ cannot intersect either the geodesic starting at $\boldsymbol{v}_{1}^{l_{2}}$ or the geodesic starting at $\boldsymbol{v}_{2}^{l_{2}}$. This is so because otherwise (by planarity) the geodesic starting at $\boldsymbol{v}_{1}^{l_{1}}$ would intersect the geodesic starting at $\boldsymbol{v}_{2}^{l_{1}}$, which contradicts the definition of $A^{l_{1}}$. Thus,

$$
A^{l_{1}} \cap A^{l_{2}} \subseteq A_{\delta}\left(\boldsymbol{x}_{1}^{l_{1}}, \boldsymbol{x}_{1}^{l_{2}}, \boldsymbol{x}_{2}^{l_{2}}, \tilde{\boldsymbol{x}}_{1}^{l_{1}}, \tilde{\boldsymbol{x}}_{1}^{l_{2}}, \tilde{\boldsymbol{x}}_{2}^{l_{2}}\right),
$$

which yields Assertion 4.1.

For $m, k \geq 0$, let $F_{m, k}$ be the event that some tree in $\delta(0)$ touches a vertex in the rectangle

$$
\boldsymbol{R}_{m, k}:=\{(x(1), x(2)): 0 \leq x(1) \leq m \text { and }|x(2)| \leq k\},
$$

but no vertex in

$$
\boldsymbol{Q}_{m}:=\{(x(1), x(2)): x(1) \leq m\} \backslash \boldsymbol{R}_{m, k} .
$$

The second step is given by the following assertion.

Assertion 4.2. If, for some $\delta \in \mathbb{Q}$ and $\boldsymbol{x}_{i}, \tilde{\boldsymbol{x}}_{i} \in \mathbb{Q}^{2}, i=1,2,3$, we have

$$
\mathrm{P}\left(A_{\delta}\left(\boldsymbol{x}_{1}, \boldsymbol{x}_{2}, \boldsymbol{x}_{3}, \tilde{\boldsymbol{x}}_{1}, \tilde{\boldsymbol{x}}_{2}, \tilde{\boldsymbol{x}}_{3}\right)\right)>0,
$$

then

$$
\mathrm{P}\left(F_{m, k}\right)>0
$$

for some $m, k \geq 0$. 
Proof. To prove this we shall use a local modification argument based on Lemma 3.1, and divide the proof into two parts: in the first one we will assume that $\mathbb{F}$ has unbounded support while in the second one we will assume that $\mathbb{F}$ has bounded support.

Part 1: $\mathbb{F}$ has unbounded support. Let $\delta \in \mathbb{Q}$ and $\boldsymbol{x}_{1}, \boldsymbol{x}_{2}, \boldsymbol{x}_{3}, \tilde{\boldsymbol{x}}_{1}, \tilde{\boldsymbol{x}}_{2}, \tilde{\boldsymbol{x}}_{3} \in \mathbb{Q}^{2}$ be the variables in the statement of Assertion 4.1. Let $\boldsymbol{R}_{0}:=[-\delta, \delta] \times\left[c_{\mathrm{s}}-\delta, c_{\mathrm{n}}+\delta\right]$, where $c_{\mathrm{n}}$ is the maximum of the second coordinates of $\boldsymbol{x}_{3}$ and $\tilde{\boldsymbol{x}}_{3}$ and $c_{\mathrm{S}}$ is the minimum of the second coordinates of $\boldsymbol{x}_{1}$ and $\tilde{\boldsymbol{x}}_{1}$. Denote by $\boldsymbol{\Xi}$ the set of edges which cross both the rectangle $\boldsymbol{R}_{0}$ and the vertical coordinate axis. Then $\boldsymbol{e}_{i}:=\left(\boldsymbol{v}_{i}, \tilde{\boldsymbol{v}}_{i}\right) \in \Xi$ for all configurations in $A_{\delta}\left(\boldsymbol{x}_{1}, \boldsymbol{x}_{2}, \boldsymbol{x}_{3}, \tilde{\boldsymbol{x}}_{1}, \tilde{\boldsymbol{x}}_{2}, \tilde{\boldsymbol{x}}_{3}\right)$ (recall that $\boldsymbol{x}_{i} \in \boldsymbol{C}_{\boldsymbol{v}_{i}}$ and $\tilde{\boldsymbol{x}}_{i} \in \boldsymbol{C}_{\tilde{\boldsymbol{v}}_{i}}$ ).

Define the event $B_{\lambda}$ as comprising those configurations such that, for all $\boldsymbol{e}=\left(\boldsymbol{v}_{1}, \boldsymbol{v}_{2}\right) \in \Xi$, there exists a $\gamma$ that connects $\boldsymbol{v}_{1}$ and $\boldsymbol{v}_{2}$ without using edges in $\Xi$ and has $t(\gamma)<\lambda$. Since

$$
\lim _{\lambda \rightarrow \infty} \mathrm{P}\left(B_{\lambda}\right)=1,
$$

we can choose a $\lambda>0$ sufficiently large that

$$
\mathrm{P}\left(A_{\delta}\left(\boldsymbol{x}_{1}, \boldsymbol{x}_{2}, \boldsymbol{x}_{3}, \tilde{\boldsymbol{x}}_{1}, \tilde{\boldsymbol{x}}_{2}, \tilde{\boldsymbol{x}}_{3}\right) \cap B_{\lambda}\right)>0 .
$$

Now we apply Lemma 3.1. To do so, we define $W(\omega)$, a random element of $K$, using the following procedure: given $\omega \in \Omega$, set

$$
W(\omega):=\left(\left(k_{j}, l_{j}, n_{j}, m_{j}\right)\right)_{j=1, \ldots, q}
$$

by ordering all $(k, l, m, n)$ (according to (3.1)) so that $\boldsymbol{e}(\omega)=\left(U_{k, l}(\omega), U_{m, n}(\omega)\right) \in \Xi(\omega)$ and $\tau_{\boldsymbol{e}} \leq \lambda$. Thus, $W$ is an ordered representation of the indices of the edges $\boldsymbol{e} \in \boldsymbol{\Xi}$ with $\tau_{\boldsymbol{e}} \leq \lambda$.

For each $I \in K$, let $R_{I}:=(\lambda, \infty)^{q} \subseteq \Omega_{I}=\mathbb{R}^{q}$, and let

$$
A:=A_{\delta}\left(x_{1}, x_{2}, x_{3}, \tilde{x}_{1}, \tilde{x}_{2}, \tilde{x}_{3}\right) \cap B_{\lambda}
$$

(given by (4.8)). Since $\mathbb{F}$ has unbounded support, $\mathrm{P}_{I}\left(R_{I}\right)>0$ for all $I \in K$. By Lemma 3.1, there exists a measurable set $\Phi(A) \subseteq \tilde{\Phi}(A)$.

Now consider a configuration $\tilde{\omega} \in \Phi(A) \subseteq \tilde{\Phi}(A)$. By definition, there exist $I \in K$, $\omega_{1} \in \hat{\Omega}_{I}, \omega_{2} \in \Omega_{I}$, and $\tilde{\omega}_{2} \in R_{I}$ such that $\tilde{\omega}=\left(\omega_{1}, \tilde{\omega}_{2}\right)$ and $\left(\omega_{1}, \omega_{2}\right) \in A$. Since $\omega_{2}$ and $\tilde{\omega}_{2}$ concern only travel times which are associated with $I$ and $\omega_{2} \leq \tilde{\omega}_{2}$ (in the canonical order in $\mathbb{R}^{q}$ ), the semi-infinite geodesics $\rho_{\tilde{v}_{i}}(0)\left(\omega_{1}, \omega_{2}\right), i=1,2,3$ (with respect to the configuration $\left(\omega_{1}, \omega_{2}\right)$ ), remain disjoint geodesics, with common asymptotic orientation $\vec{e}_{1}$, for the configuration $\tilde{\omega}=\left(\omega_{1}, \tilde{\omega}_{2}\right)$. For the same reason, $\tilde{\omega} \in B_{\lambda}$. On the other hand, since $\tilde{\omega}_{2} \in R_{I}$, we have $\tau_{\boldsymbol{e}}(\tilde{\omega})>\lambda$ for all $\boldsymbol{e} \in \Xi$ and, thus, no geodesic could have an edge in $\Xi$. Therefore, $\Phi(A) \subseteq F_{m, k}$, where $k:=\max \left\{c_{\mathrm{s}}, c_{\mathrm{n}}\right\}$ and $m:=\delta+\max \left\{\tilde{x}_{1}(1), \tilde{x}_{2}(1), \tilde{x}_{3}(1)\right\}$. Since $\mathrm{P}(A)>0$, we also have $0<\mathrm{P}(\Phi(A)) \leq \mathrm{P}\left(F_{m, k}\right)$, which yields Assertion 4.2 when $\mathbb{F}$ has unbounded support.

Part 2: $\mathbb{F}$ has bounded support. Again let $\delta \in \mathbb{Q}$ and $\boldsymbol{x}_{1}, \boldsymbol{x}_{2}, \boldsymbol{x}_{3}, \tilde{\boldsymbol{x}}_{1}, \tilde{\boldsymbol{x}}_{2}, \tilde{\boldsymbol{x}}_{3} \in \mathbb{Q}^{2}$ be as in Assertion 4.1. Let $\vec{e}_{2}:=(0,1), \boldsymbol{c}_{\mathrm{n}}:=\left(0, c_{\mathrm{n}}\right)$, and $\boldsymbol{c}_{\mathrm{S}}:=\left(0, c_{\mathrm{S}}\right)$. For $\varepsilon, \tilde{\varepsilon}>0$ and $m>0$, let

$$
\boldsymbol{Q}_{m, \tilde{\varepsilon}}:=m \vec{e}_{1}+\left[-\tilde{\varepsilon} m \vec{e}_{2}, \tilde{\varepsilon} \vec{e}_{2}\right]
$$

and let $B_{m}^{\varepsilon, \tilde{\varepsilon}}$ be the event that, for every $\boldsymbol{z} \in\left[\boldsymbol{c}_{\mathrm{s}}, \boldsymbol{c}_{\mathrm{n}}\right]$ and every $\boldsymbol{u} \in \boldsymbol{Q}_{m, \tilde{\varepsilon}}$,

$$
T(z, \boldsymbol{u})<(\mu+\varepsilon) m .
$$


By the shape theorem, for any $\varepsilon>0$ and for sufficiently small $\tilde{\varepsilon}$ we have

$$
\lim _{m \rightarrow \infty} \mathrm{P}\left(B_{m}^{\varepsilon, \tilde{\varepsilon}}\right)=1 .
$$

Denote by $C_{m}^{\tilde{\varepsilon}}$ the event that, for each $i=1,2,3, \rho_{\boldsymbol{v}_{i}}(0)$ touches the hyperplane that has direction $\vec{e}_{2}$ and contains $(0, m)$ for the first time (coming from $\boldsymbol{v}_{i}$ ) within the vertical segment $\boldsymbol{Q}_{m, \tilde{\varepsilon}}$. Since all those geodesics are 0-paths,

$$
\lim _{m \rightarrow \infty} \mathrm{P}\left(C_{m}^{\tilde{\varepsilon}}\right)=1
$$

for all $\tilde{\varepsilon}>0$.

For $m, k>0$, let $C_{m, k}$ denote the event that, for each $i=1,2,3, \rho_{\boldsymbol{v}_{i}}(0)$ does not intersect the region consisting of the points $(x(1), x(2)) \in \mathbb{R}^{2}$ with $x(1) \in[0, m]$ and $|x(2)|>k$. Thus, for any fixed $m>0$,

$$
\lim _{k \rightarrow \infty} \mathrm{P}\left(C_{m, k}\right)=1
$$

(for the same reason (4.10) holds).

Let $\boldsymbol{x}, \boldsymbol{y} \in \mathbb{R}^{2}$ and recall the definition of the path $\gamma(\boldsymbol{x}, \boldsymbol{y})$ given in Subsection 3.2. By Lemma 3.2,

$$
\lim _{n \rightarrow \infty} \mathrm{P}\left(\left|\gamma\left(\mathbf{0}, n \vec{e}_{1}\right)\right| \geq c_{1} n\right)=0
$$

for some constant $c_{1}>0$. We also introduced the graph metric $T_{\mathscr{D}}$; by Lemma 3.3, we have

$$
\lim _{n \rightarrow \infty} \frac{T_{\mathscr{D}}\left(\left[\boldsymbol{c}_{\mathrm{s}}, \boldsymbol{c}_{\mathrm{n}}\right], \boldsymbol{Q}_{m, \tilde{\varepsilon}}\right)}{m}=v .
$$

For each $i=1,2,3$, let $\rho_{i}$ denote the piece of $\rho_{\boldsymbol{v}_{i}}(0)$ between $\tilde{\boldsymbol{v}}_{i}$ and the first point, say $\boldsymbol{u}_{i}$, at which it intersects $\left[m \vec{e}_{1}-\tilde{\varepsilon} m \vec{e}_{2}, m \vec{e}_{1}+\tilde{\varepsilon} m \vec{e}_{2}\right]$. For $z \in\left[\boldsymbol{c}_{\mathrm{s}}, \boldsymbol{c}_{\mathrm{n}}\right]$ and $\boldsymbol{u} \in \boldsymbol{Q}_{m, \tilde{\varepsilon}}$, let $\phi(z, \boldsymbol{u})$ be the path connecting $z$ and $\boldsymbol{u}$ which first moves vertically along $\gamma\left(\boldsymbol{z}, \boldsymbol{v}_{1}\right)$, then follows $\rho_{1}$, and then moves vertically again along $\gamma\left(\boldsymbol{u}_{1}, \boldsymbol{u}\right)$. Thus, on the intersection between $A_{\delta}\left(\boldsymbol{x}_{1}, \boldsymbol{x}_{2}, \boldsymbol{x}_{3}, \tilde{\boldsymbol{x}}_{1}, \tilde{\boldsymbol{x}}_{2}, \tilde{\boldsymbol{x}}_{3}\right), C_{m}^{\tilde{\varepsilon}}, C_{m, k}$, and $B_{m}^{\varepsilon, \tilde{\varepsilon}}$, we have

$$
\begin{aligned}
t(\phi(z, \boldsymbol{u})) & =t\left(\gamma\left(\boldsymbol{z}, \boldsymbol{v}_{1}\right)\right)+t\left(\rho_{1}\right)+t\left(\gamma\left(\boldsymbol{u}_{1}, \boldsymbol{u}\right)\right) \\
& \leq \lambda\left|\gamma\left(\boldsymbol{c}_{\mathrm{s}}, \boldsymbol{c}_{\mathrm{n}}\right)\right|+(\mu+\varepsilon) m+\lambda\left|\gamma\left(m \vec{e}_{1}-\tilde{\varepsilon} m \vec{e}_{2}, m \vec{e}_{1}+\tilde{\varepsilon} m \vec{e}_{2}\right)\right| .
\end{aligned}
$$

Also, by (4.12) and (4.13), since $\mu(\mathbb{F})<\lambda(\mathbb{F}) v$ it follows that there exist $\varepsilon_{0}, \tilde{\varepsilon}_{0}>0$ such that

$$
\lim _{m \rightarrow \infty} \mathrm{P}(D(\lambda, \varepsilon, \tilde{\varepsilon}))=1
$$

for all $\varepsilon<\varepsilon_{0}$ and $\tilde{\varepsilon}<\tilde{\varepsilon}_{0}$, where $D(\lambda, \varepsilon, \tilde{\varepsilon})$ is the event that

$$
\lambda\left|\gamma\left(\boldsymbol{c}_{\mathrm{s}}, \boldsymbol{c}_{\mathrm{n}}\right)\right|+(\mu+\varepsilon) m+\lambda\left|\gamma\left(m \vec{e}_{1}-\tilde{\varepsilon} m \vec{e}_{2}, m \vec{e}_{1}+\tilde{\varepsilon} m \vec{e}_{2}\right)\right| \leq(\lambda-\varepsilon) T_{\mathscr{D}}\left(\left[\boldsymbol{c}_{\mathrm{s}}, \boldsymbol{c}_{\mathrm{n}}\right], \boldsymbol{Q}_{m, \tilde{\varepsilon}}\right) .
$$

Let

$$
A:=A_{\delta}\left(\boldsymbol{x}_{1}, \boldsymbol{x}_{2}, \boldsymbol{x}_{3}, \tilde{\boldsymbol{x}}_{1}, \tilde{\boldsymbol{x}}_{2}, \tilde{\boldsymbol{x}}_{3}\right) \cap C_{m}^{\tilde{\varepsilon}} \cap C_{m, k} \cap B_{m}^{\varepsilon, \tilde{\varepsilon}} \cap D(\lambda, \varepsilon, \tilde{\varepsilon}) .
$$

Combining (4.9) with (4.10), (4.11), and (4.14), we find that $\mathrm{P}(A)>0$ for sufficiently small $\varepsilon>0$ and $\tilde{\varepsilon}>0$ and for sufficiently large $m>0$ and $k>0$. Note that, for all configurations in $A$ and every $\boldsymbol{z} \in\left[\boldsymbol{c}_{\mathrm{s}}, \boldsymbol{c}_{\mathrm{n}}\right]$ and $\boldsymbol{u} \in \boldsymbol{Q}_{m, \tilde{\varepsilon}}$, we must have

$$
T(\boldsymbol{z}, \boldsymbol{u}) \leq t(\phi(z, \boldsymbol{u})) \leq(\lambda-\varepsilon) T_{\mathscr{D}}\left(\left[\boldsymbol{c}_{\mathrm{s}}, \boldsymbol{c}_{\mathrm{n}}\right], \boldsymbol{Q}_{m, \tilde{\varepsilon}}\right) .
$$


We can now use Lemma 3.1 again. Let $\Xi$ be the set of edges in the interior of the region bounded by $\rho_{1}, \rho_{3},\left[\boldsymbol{c}_{\mathrm{s}}, \boldsymbol{c}_{\mathrm{n}}\right]$, and $\boldsymbol{Q}_{m, \tilde{\varepsilon}}$. Define $W(\omega)$ as follows: given $\omega \in \Omega$, set

$$
W(\omega):=\left(\left(k_{j}, l_{j}, m_{j}, n_{j}\right)\right)_{j=1, \ldots, q}
$$

by ordering all $(k, l, m, n)$ (according to (3.1)) so that $\boldsymbol{e}(\omega)=\left(U_{k, l}(\omega), U_{m, n}(\omega)\right) \in \Xi(\omega)$ with $\tau_{\boldsymbol{e}} \leq \lambda-\varepsilon$. Thus, $W$ represents the indices of the edges $\boldsymbol{e} \in \boldsymbol{\Xi}$ with $\tau_{\boldsymbol{e}} \leq \lambda-\varepsilon$. For each $I \in K$, let $R_{I}:=(\lambda-\varepsilon, \lambda)^{q} \subseteq \Omega_{I}$ and take $A$ to be defined as above. Since $\mathbb{F}(\lambda-\varepsilon)<1$, we have $\mathrm{P}_{I}\left(R_{I}\right)>0$. Thus, by Lemma (3.1) there exists a measurable set $\Phi(A) \subseteq \tilde{\Phi}(A)$.

Choose a configuration $\tilde{\omega}=\left(\omega_{1}, \tilde{\omega}_{2}\right) \in \tilde{\Phi}(A)$. By the same argument used in part 1 , we see that the paths $\rho_{\tilde{\boldsymbol{v}}_{i}}(0)\left(\omega_{1}, \omega_{2}\right), i=1,2$, and $\rho_{\tilde{\boldsymbol{u}}_{2}}(0)\left(\omega_{1}, \omega_{2}\right)$ remain disjoint geodesics, with common asymptotic orientation $\vec{e}_{1}$, for the configuration $\tilde{\omega}$, and that (4.15) still holds for the configuration $\tilde{\omega}$. On the other hand, by (4.15), no path $\rho$ that connects $z \in\left[c_{\mathrm{s}}, c_{\mathrm{n}}\right]$ and $\boldsymbol{u} \in \boldsymbol{Q}_{m, \tilde{\varepsilon}}$ and is entirely contained in the region $\boldsymbol{\Xi}$ can be a geodesic for the configuration $\tilde{\omega}$ because, otherwise,

$$
T(\boldsymbol{z}, \boldsymbol{u})=t(\rho)>(\lambda-\varepsilon) T_{\mathscr{D}}\left(\left[\boldsymbol{c}_{\mathrm{s}}, \boldsymbol{c}_{\mathrm{n}}\right], \boldsymbol{Q}_{m, \tilde{\varepsilon}}\right) .
$$

This allows us to conclude that

$$
\Phi(A) \subseteq \tilde{\Phi}(A) \subseteq F_{m, k}
$$

(with $m, k>0$ given by the definition of $A$ ). Since $\mathrm{P}(A)>0$, we have $0<\mathrm{P}(\Phi(A))<$ $\mathrm{P}\left(F_{m, k}\right)$, which yields Assertion 4.2 when $\mathbb{F}$ has bounded support.

The third and last step in proving that $\mathrm{P}(N(\alpha) \leq 1)=1$ is as follows.

Assertion 4.3. $\mathrm{P}\left(F_{m, k}\right)=0$ for all $m, k \geq 0$.

Proof. Consider a rectangular array of nonintersecting translates $\boldsymbol{R}_{m, k}^{z}$ of the basic rectangle $\boldsymbol{R}_{m, k} \equiv \boldsymbol{R}_{m, k}^{\mathbf{0}}$ and an array of translates $\boldsymbol{Q}_{m}^{z}$ of $\boldsymbol{Q}_{m} \equiv \boldsymbol{Q}_{m}^{\mathbf{0}}$, indexed by $\boldsymbol{z} \in \mathbb{Z}_{\tilde{z}}^{2}$. We denote by $F_{m, k}^{z}$ the corresponding events, with $F_{m, k}^{\mathbf{0}} \equiv F_{m, k}$. Note that if $F_{m, k}^{z}$ and $F_{m, k}^{\tilde{z}}, z \neq \tilde{z}$, occur, then the corresponding trees in $\&(0)$ must be disjoint. Thus, if $N_{L}$ is the number of points $z \in[0, L]^{2}$ for which $F_{m, k}^{z}$ occurs, then

$$
N_{L} \leq \mid\left\{\text { edges crossing the boundary of }[0, L]^{2}\right\} \mid .
$$

However, by Lemma 3.2, the expected value of the number of edges crossing the boundary of $[0, L]^{2}$ is of order $L$. By translation invariance,

$$
\mathrm{E}\left(N_{L}\right)=n_{L} \mathrm{P}\left(F_{m, k}\right),
$$

where $n_{L}$ is the number of rectangles $\boldsymbol{R}_{m, k}^{z}$ intersecting $[0, L]^{2}$. Since $n_{L}$ is of order $L^{2}$, the assumption $\mathrm{P}\left(F_{m, k}\right)>0$ leads to a contradiction.

Now we are able to prove Proposition 4.3.

Proof of Proposition 4.3. Combining Assertions 4.1-4.3 yields

$$
\mathrm{P}(N(\alpha) \leq 1)=\mathrm{P}(N(0) \leq 1)=1 .
$$

By noticing that $\Omega_{3} \cap\{N(\alpha) \leq 1\} \subseteq \Omega_{4}(\alpha)$, we see that Proposition 4.3 follows from Proposition 4.2 together with (4.16). 


\subsection{Existence and asymptotics of the Busemann function}

The idea of the proof of Theorem 1.3 is to combine the properties of existence, uniqueness, and coalescence of semi-infinite geodesics in a fixed direction $\mathrm{e}^{\mathrm{i} \alpha}$ to show that if $z_{n} \rightarrow \infty$ along this direction, then, for sufficiently large $n$,

$$
T\left(\boldsymbol{x}, z_{n}\right)-T\left(\boldsymbol{y}, z_{n}\right)=T(\boldsymbol{x}, \boldsymbol{c})-T(\boldsymbol{y}, \boldsymbol{c}),
$$

where $c$ is the coalescence point in the direction $\mathrm{e}^{\mathrm{i} \alpha}$ (see Proposition 4.3). We begin by introducing what we mean by convergence of paths. Assume that $\left(\gamma_{n}\right)_{n \geq 0}$ is a sequence of finite paths with vertices in $\mathbb{R}^{2}$, and for each $n \geq 0$ let $\gamma_{n}=\left(z_{0}^{n}, z_{1}^{n}, \ldots, z_{l_{n}}^{n}\right)$. We say that $\gamma_{n}$ converges to a semi-infinite path $\gamma=\left(x_{0}, x_{1}, \ldots\right)$, and write $\gamma=\lim _{n \rightarrow \infty} \gamma_{n}$, if for each $k \geq 1$ there exists an $n_{k} \geq 1$ such that

$$
\gamma_{n}=\left(x_{0}, x_{1}, \ldots, x_{k}, z_{k+1}^{n}, \ldots, z_{l_{n}}^{n}\right) \text { for all } n \geq n_{k} .
$$

For each sequence $\left(z_{n}\right)_{n \geq 0}$ of vertices in $\mathbb{R}^{2}$ with $\left|z_{n}\right| \rightarrow \infty$ and $z \in \mathbb{R}^{2}$, we denote by $\Pi\left(z,\left(z_{n}\right)_{n \geq 0}\right)$ the set of all semi-infinite paths $\rho$ for which there exists a subsequence $\left(n_{j}\right)_{j \geq 0}$ with $\lim _{j \rightarrow \infty} \rho\left(z, z_{n_{j}}\right)=\rho$.

Lemma 4.3. Let $\Omega_{1}$ be the event that, for all $\alpha \in[0,2 \pi)$, if $\left(z_{n}\right)_{n \geq 1}$ has asymptotic orientation $\mathrm{e}^{\mathrm{i} \alpha}$ then (i) $\Pi\left(z,\left(z_{n}\right)_{n \geq 1}\right) \neq \varnothing$ and (ii) every $\rho \in \Pi\left(z,\left(z_{n}\right)_{n \geq 1}\right)$ is a semi-infinite geodesic with asymptotic orientation $\mathrm{e}^{\mathrm{i} \alpha}$. Under (1.1), we then have $\mathrm{P}\left(\Omega_{1}\right)=1$.

Proof. Let $\mathcal{T}$ be the tree with vertex set $\bigcup_{n \geq 1} \rho\left(\boldsymbol{z}, \boldsymbol{z}_{n}\right)$ and oriented edges $(\boldsymbol{u}, \boldsymbol{v}) \in \mathscr{D}_{\mathrm{e}}$ (in the Delaunay triangulation) such that $\rho(z, \boldsymbol{u}) \subseteq \rho(\boldsymbol{z}, \boldsymbol{v})$. Note that $\mathcal{T}$ is an infinite tree. Since every vertex in the Delaunay triangulation has finite degree, the same is true of the vertices of $\mathcal{T}$. Therefore, by a standard compactness argument, $\Pi\left(z,\left(z_{n}\right)_{n \geq 1}\right) \neq \varnothing$. To show that every $\rho \in \Pi\left(\left(z_{n}\right)_{n \geq 1}\right)$ has the asymptotic orientation $\mathrm{e}^{\mathrm{i} \alpha}$, consider the set $\boldsymbol{D} \subseteq \boldsymbol{S}^{1}$ defined in the proof of Theorem 1.1. By Proposition 4.1 and Proposition 4.2, for all $\beta \in[0,2 \pi)$ such that $\mathrm{e}^{\mathrm{i} \beta} \in \boldsymbol{D}$ there almost surely exists a unique semi-infinite geodesic that starts at $\boldsymbol{v}(\boldsymbol{z})$ and has asymptotic orientation $\mathrm{e}^{\mathrm{i} \beta}$, which we denote by $\rho_{z}(\beta)$. Now, let $\beta_{1}, \beta_{2} \in[0,2 \pi)$ be such that $\mathrm{e}^{\mathrm{i} \beta_{1}}, \mathrm{e}^{\mathrm{i} \beta_{2}} \in \boldsymbol{D}$. Assume further that, by following the counterclockwise orientation of $\boldsymbol{S}^{1}$, the unit vector $\mathrm{e}^{\mathrm{i} \alpha}$ lies between the unit vectors $\mathrm{e}^{\mathrm{i} \beta_{1}}$ and $\mathrm{e}^{\mathrm{i} \beta_{2}}$. Note that the paths $\rho_{z}\left(\beta_{1}\right)$ and $\rho_{z}\left(\beta_{2}\right)$ bifurcate at some point $\boldsymbol{v}$ and have no further points in common. On the other hand, $\left(z_{n}\right)_{n \geq 0}$ has the asymptotic orientation $\mathrm{e}^{\mathrm{i} \alpha}$. Therefore, for large enough $k, \rho\left(z, z_{k}\right)$ should lie between $\rho_{z}\left(\beta_{1}\right)$ and $\rho_{z}\left(\beta_{2}\right)$, and, thus, the same is true for any limit $\rho$. Since $\boldsymbol{D}$ is dense in $\boldsymbol{S}^{1}$, it follows that $\rho$ has the asymptotic orientation $\mathrm{e}^{\mathrm{i} \alpha}$.

Proof of Theorem 1.3. Consider the intersection between $\Omega_{1}$ (the event of path convergence (see Lemma 4.3)) and $\Omega_{4}(\alpha)$ (the event of coalescence and uniqueness of semi-infinite geodesics (see Proposition 4.3)). On this event, if $\left(z_{n}\right)_{n \geq 1}$ has the asymptotic orientation $\mathrm{e}^{\mathrm{i} \alpha}$, then $\lim _{n \rightarrow \infty} \rho\left(\boldsymbol{x}, \boldsymbol{z}_{n}\right)=\rho_{\boldsymbol{x}}(\alpha)$. Together with coalescence, this implies that for $\boldsymbol{x}, \boldsymbol{y} \in \mathbb{R}^{2}$ there exist $\boldsymbol{c} \equiv \boldsymbol{c}(\boldsymbol{x}, \boldsymbol{y}, \alpha) \in \mathcal{D}_{\mathrm{v}}$ and $n_{0}>0$ such that

$$
\rho\left(\boldsymbol{x}, \boldsymbol{z}_{n}\right)=\rho(\boldsymbol{x}, \boldsymbol{c}) \cup \rho\left(\boldsymbol{c}, \boldsymbol{z}_{n}\right) \quad \text { and } \quad \rho\left(\boldsymbol{y}, \boldsymbol{z}_{n}\right)=\rho(\boldsymbol{y}, \boldsymbol{c}) \cup \rho\left(\boldsymbol{c}, \boldsymbol{z}_{n}\right)
$$

for all $n \geq n_{0}$, which implies that

$$
T\left(\boldsymbol{x}, z_{n}\right)-T\left(\boldsymbol{y}, z_{n}\right)=T(\boldsymbol{x}, \boldsymbol{c})-T(\boldsymbol{y}, \boldsymbol{c})
$$

for all $n \geq n_{0}$. 
Proof of Theorem 1.4. Let $\mathbb{H}_{r}^{\alpha}$ be the hyperplane that passes through $\boldsymbol{a}_{r}:=a_{r} \mathrm{e}^{\mathrm{i} \alpha}$ and $r \vec{e}_{1}$, where $a_{r}=r / \cos \alpha$. Let $\boldsymbol{x}_{r}$ be the crossing point of the linear interpolation of $\rho_{\mathbf{0}}(\alpha)$ and $\mathbb{H}_{r}^{\alpha}$ of maximal distance from $\boldsymbol{a}_{r}$. We claim that

$$
-T\left(r \vec{e}_{1}, \mathbf{0}\right) \leq H^{\alpha}\left(r \vec{e}_{1}, \mathbf{0}\right) \leq T\left(r \vec{e}_{1}, \boldsymbol{x}_{r}\right)-T\left(\boldsymbol{x}_{r}, \mathbf{0}\right) .
$$

The first inequality in (4.17) follows directly from the triangle inequality for $T$, since $H^{\alpha}\left(r \vec{e}_{1}, \mathbf{0}\right)=T\left(r \vec{e}_{1}, \boldsymbol{c}_{r}\right)-T\left(\mathbf{0}, \boldsymbol{c}_{r}\right)$ (as in the proof of Theorem 1.3). To prove the second inequality, notice that if $\boldsymbol{x}_{r} \notin \rho\left(\mathbf{0}, \boldsymbol{c}_{r}\right)$ then $\boldsymbol{c}_{r} \in \rho\left(\mathbf{0}, \boldsymbol{x}_{r}\right)$, which implies that $\boldsymbol{c}_{r} \in \rho\left(r \vec{e}_{1}, \boldsymbol{x}_{r}\right)$. Thus,

$$
H^{\alpha}\left(r \vec{e}_{1}, \mathbf{0}\right)=T\left(r \vec{e}_{1}, \boldsymbol{c}_{r}\right)-T\left(\mathbf{0}, \boldsymbol{c}_{r}\right)=T\left(r \vec{e}_{1}, \boldsymbol{x}_{r}\right)-T\left(\boldsymbol{x}_{r}, \mathbf{0}\right) .
$$

If $\boldsymbol{x}_{r} \in \rho\left(\boldsymbol{0}, \boldsymbol{c}_{r}\right)$ then

$$
T\left(\mathbf{0}, \boldsymbol{c}_{r}\right)=T\left(\mathbf{0}, \boldsymbol{x}_{r}\right)+T\left(\boldsymbol{x}_{r}, \boldsymbol{c}_{r}\right)
$$

Consequently,

$$
H^{\alpha}\left(r \vec{e}_{1}, \mathbf{0}\right)=T\left(r \vec{e}_{1}, \boldsymbol{c}_{r}\right)-T\left(\mathbf{0}, \boldsymbol{c}_{r}\right)=T\left(r \vec{e}_{1}, \boldsymbol{c}_{r}\right)-T\left(\boldsymbol{c}_{r}, \boldsymbol{x}_{r}\right)-T\left(\mathbf{0}, \boldsymbol{x}_{r}\right) .
$$

Since (again from the triangle inequality)

$$
T\left(r \vec{e}_{1}, c_{r}\right)-T\left(c_{r}, x_{r}\right) \leq T\left(r \vec{e}_{1}, x_{r}\right),
$$

(4.18) yields (4.17).

Now,

$$
\begin{aligned}
T\left(r \vec{e}_{1}, \boldsymbol{x}_{r}\right)-T\left(\boldsymbol{x}_{r}, \mathbf{0}\right)= & \left(T\left(r \vec{e}_{1}, \boldsymbol{x}_{r}\right)-\mu\left|r \vec{e}_{1}-\boldsymbol{a}_{r}\right|\right)+\left(\mu\left|\boldsymbol{a}_{r}\right|-T\left(\boldsymbol{x}_{r}, \mathbf{0}\right)\right) \\
& +\left(\mu\left|r \vec{e}_{1}-\boldsymbol{a}_{r}\right|-\mu\left|\boldsymbol{a}_{r}\right|\right) \\
=: & \left(I_{1}(r)\right)+\left(I_{2}(r)\right)+\left(I_{3}(r)\right) .
\end{aligned}
$$

According to Remark 4.1, if we choose $\xi \in\left(\frac{3}{4}, 1\right)$ then, for some constant $c>0,\left|\boldsymbol{x}_{r}-\boldsymbol{a}_{r}\right| \leq c r^{\xi}$ almost surely for sufficiently large $r$. On the other hand, by the triangle inequality,

$$
\begin{aligned}
\left|T\left(\boldsymbol{x}_{r}, r \vec{e}_{1}\right)-T\left(\boldsymbol{a}_{r}, r \vec{e}_{1}\right)\right| & \leq T\left(\boldsymbol{x}_{r}, \boldsymbol{a}_{r}\right), \\
\left|T\left(\boldsymbol{x}_{r}, \mathbf{0}\right)-T\left(\boldsymbol{a}_{r}, \mathbf{0}\right)\right| & \leq T\left(\boldsymbol{x}_{r}, \boldsymbol{a}_{r}\right) .
\end{aligned}
$$

Thus,

$$
\begin{array}{r}
\limsup _{r \rightarrow \infty} \frac{\left|I_{1}(r)\right|}{r} \leq \limsup _{r \rightarrow \infty} \frac{\left|T\left(r \vec{e}_{1}, \boldsymbol{a}_{r}\right)-\mu\right| r \vec{e}_{1}-\boldsymbol{a}_{r}||}{r} \\
+\limsup _{r \rightarrow \infty} \frac{\max _{\left|z-\boldsymbol{a}_{r}\right| \leq c r^{\xi}}\left\{T\left(\boldsymbol{a}_{r}, \boldsymbol{z}\right)\right\}}{r}
\end{array}
$$

and

$$
\begin{aligned}
\limsup _{r \rightarrow \infty} \frac{\left|I_{2}(r)\right|}{r} \leq & \limsup _{r \rightarrow \infty} \frac{\left|T\left(\mathbf{0}, \boldsymbol{a}_{r}\right)-\mu\right| \boldsymbol{a}_{r}||}{r} \\
& +\limsup _{r \rightarrow \infty} \frac{\max _{\left|z-\boldsymbol{a}_{r}\right| \leq c r^{\xi}}\left\{T\left(\boldsymbol{a}_{r}, \boldsymbol{z}\right)\right\}}{r} .
\end{aligned}
$$


Combining Lemma 4.1 with translation invariance, we find that, for all $\varepsilon>0$,

$$
\begin{array}{r}
\sum_{r \geq 1} \mathrm{P}\left(\left|T\left(r \vec{e}_{1}, \boldsymbol{a}_{r}\right)-\mu\right| r \vec{e}_{1}-\boldsymbol{a}_{r}|| \geq \varepsilon r\right)<\infty, \\
\sum_{r \geq 1} \mathrm{P}\left(\left|T\left(\mathbf{0}, \boldsymbol{a}_{r}\right)-\mu\right| \boldsymbol{a}_{r}|| \geq \varepsilon r\right)<\infty .
\end{array}
$$

Therefore, by the Borel-Cantelli lemma,

$$
\begin{array}{r}
\limsup _{r \rightarrow \infty} \frac{\left|T\left(r \vec{e}_{1}, \boldsymbol{a}_{r}\right)-\mu\right| r \vec{e}_{1}-\boldsymbol{a}_{r}||}{r}=0, \\
\limsup _{r \rightarrow \infty} \frac{\left|T\left(\mathbf{0}, \boldsymbol{a}_{r}\right)-\mu\right| \boldsymbol{a}_{r}||}{r}=0 .
\end{array}
$$

In Lemma 4.3 of [17] it was proved that, for some constants $c_{0}, x_{0}>0$, if $x>x_{0}$ then

$$
\mathrm{P}(T(\mathbf{0}, z)>x|z|) \leq \mathrm{e}^{-c_{0} x|z|} .
$$

By noting that, with high probability, the number of vertices belonging to a ball of radius $c r^{\xi}$ is of order $r^{2 \xi}$, we find that, for all $\varepsilon>0$,

$$
\sum_{r \geq 1} \mathrm{P}\left(\max _{|z| \leq c r^{\xi}}\{T(\mathbf{0}, z)\}>\varepsilon r\right)<\infty .
$$

Thus, together with the Borel-Cantelli lemma (and translation invariance), this yields

$$
\limsup _{r \rightarrow \infty} \frac{\max _{\left|z-\boldsymbol{a}_{r}\right| \leq c r}\left\{T\left(\boldsymbol{a}_{r}, \boldsymbol{z}\right)\right\}}{r}=0 .
$$

Consequently,

$$
\limsup _{r \rightarrow \infty} \frac{\left|I_{1}(r)\right|}{r}=\limsup _{r} \frac{\left|I_{2}(r)\right|}{r}=0 .
$$

Since

$$
\lim _{r \rightarrow \infty} \frac{I_{3}(r)}{r}=\mu \frac{\sin \alpha-1}{\cos \alpha}=-\mu \frac{\cos \alpha}{1+\sin \alpha},
$$

we finally obtain

$$
\lim _{r \rightarrow \infty} \frac{T\left(r \vec{e}_{1}, \boldsymbol{x}_{r}\right)-T\left(\boldsymbol{x}_{r}, \mathbf{0}\right)}{r}=-\mu \frac{\cos \alpha}{1+\sin \alpha} .
$$

Together with (4.17), this yields Theorem 1.4.

\subsection{Competition versus coalescence}

In this section we give a sketch of the proof of the statements in Remark 1.1. Let $\varphi:=$ $\left(z_{1}, z_{2}, \ldots\right)$ be a branch of the competition interface. This branch thus marks the boundary between two different species, say $j_{1}$ and $j_{2}$. Assume further that if one moves along $z_{n}, z_{n+1}, \ldots$, then on the right-hand side we always see species $j_{1}$, while on the left-hand side we see species $j_{2}$. By Theorem 1.1, this branch has the direction $\mathrm{e}^{\mathrm{i} \theta}$ for some $\theta \equiv \theta(\varphi)$. For $l=1,2$, let $\left(\boldsymbol{v}_{n}^{l}\right)_{n \geq 1}$ be the sequence of vertices in $\mathcal{D}_{\mathrm{v}} \cap \boldsymbol{B}_{\boldsymbol{x}_{j_{l}}}$ such that the tile $\boldsymbol{C}_{\boldsymbol{v}_{n}^{l}}$ has an edge boundary that belongs to $\varphi$. Thus, $\boldsymbol{v}_{n}^{l}$ has the asymptotic orientation $\mathrm{e}^{\mathrm{i} \theta(\varphi)}$ (since, by Lemma 3.4, the distance between $\boldsymbol{v}_{n}^{l}$ and the corresponding branch of the competition interface 
is small in comparison with $\left.\left|\boldsymbol{v}_{n}\right|\right)$. Together with Lemma 4.3, this implies that there exist a subsequence $\left(n_{m}\right)_{m \geq 1}$ and a semi-infinite geodesic $\rho_{l}$, with asymptotic orientation $\theta(\varphi)$, such that $\rho\left(\boldsymbol{x}_{l}, \boldsymbol{v}_{n_{m}}^{l}\right) \rightarrow \rho_{l}$. Since $\rho\left(\boldsymbol{x}_{l}, \boldsymbol{v}_{n}^{l}\right)$ is a geodesic connecting two points in $\boldsymbol{B}_{\boldsymbol{x}_{j_{l}}}(\infty)$, it follows that $\rho\left(\boldsymbol{x}_{l}, \boldsymbol{v}_{n}^{l}\right) \subseteq \boldsymbol{B}_{\boldsymbol{x}_{j_{l}}}(\infty)$ and, thus, that $\rho_{l} \subseteq \boldsymbol{B}_{\boldsymbol{x}_{j_{l}}}(\infty)$.

Consequently, we have two geodesics, $\rho_{1}$ and $\rho_{2}$, that have the same orientation, $\mathrm{e}^{\mathrm{i} \theta(\varphi)}$, but do not coalesce (because $\rho_{i} \subseteq \boldsymbol{B}_{\boldsymbol{x}_{j_{l}}}$ for $l=1,2$ ). By Proposition 4.3, this occurs with zero probability, which proves the first statement of Remark 1.1.

By Remark 4.1, for all $\xi \in\left(\frac{3}{4}, 1\right), \rho_{1}$ and $\rho_{2}$ are $(1-\xi)$-straight about their common asymptotic orientation, $\mathrm{e}^{\mathrm{i} \theta(\varphi)}$. Since $\varphi$ is confined between $\rho_{1}$ and $\rho_{2}$, this also implies that $\varphi$ is $(1-\xi)$-straight about the asymptotic orientation $\mathrm{e}^{\mathrm{i} \theta(\varphi)}$, which proves the second statement of Remark 1.1.

\section{Acknowledgements}

This work was developed during my doctoral studies (see [16]) at IMPA and I would like to thank my adviser, Professor Vladas Sidoravicius, for his dedication and encouragement during this period. I also thank Professor Charles Newman for proposing to me the problem studied here, Professor Thomas Mountford for a careful reading of, and useful comments about, a previous version of this work, and Professor James Martin for providing me with the numerical simulations whose results are shown in Figure 1. Finally, I thank the whole administrative staff of IMPA for their assistance and CNPQ for financing my doctoral studies, without which this work would have not been possible.

\section{References}

[1] Burton, R. and Keane, M. (1989). Density and uniqueness in percolation. Commun. Math. Phys. 121,501-505.

[2] Deijfen, M., Häggström, O. and Bagley, J. (2004). A stochastic model for competing growth on $\mathbf{R}^{d}$. Markov Process. Relat. Fields 10, 217-248.

[3] Derrida, B. And Dickman, R. (1991). On the interface between two growing Eden clusters. J. Phys. A 24, 191-193.

[4] Ferrari, P. A. and Pimentel, L. P. R. (2005). Competition interfaces and second class particles. Ann. Prob. 33, 1235-1254.

[5] Ferrari, P. A., Martin, J. B. and Pimentel, L. P. R. (2006). Roughening and inclination of competition interfaces. Phys. Rev. E 73, 031602.

[6] Garet, O. and Marchand, R. (2005). Coexistence in two-type first-passage percolation models. Ann. Appl. Prob. 15, 298-330.

[7] Häggström, O. and Pemantle, R. (1998). First passage percolation and a model for competing spatial growth. J. Appl. Prob. 35, 683-692.

[8] Häggström, O. and Pemantle, R. (1999). Absence of mutual unbounded growth for almost all parameter values in the two-type Richardson model. Stoch. Process. Appl. 90, 207-222.

[9] Hammersley, J. M. and Welsh, D. J. A. (1965). First-passage percolation, subadditive processes, stochastic networks, and generalized renewal theory. In Proc. Internat. Res. Semin., Springer, New York, pp. 61-110.

[10] Hoffmann, C. (2005). Coexistence for Richardson type competing spatial growth models. Ann. Appl. Prob. 15, 739-747.

[11] Howard, C. D. and Newman, C. M. (1997). Euclidean models of first-passage percolation. Prob. Theory Relat. Fields 108, 153-170.

[12] Howard, C. D. and Newman, C. M. (2001). Geodesics and spanning trees for Euclidean first-passage percolation. Ann. Prob. 29, 577-623.

[13] Licea, C. and Newman, C. M. (1996). Geodesics in two-dimensional first-passage percolation. Ann. Prob. 24, 399-410.

[14] Newman, C. M. (1995). A surface view of first-passage percolation. In Proc. Internat. Congr. Mathematicians (Zürich, 1994), ed. S. D. Chatterji, Birkhäuser, Basel, pp. 1017-1023.

[15] Newman, C. M. And Piza, M. S. T. (1995). Divergence of shape fluctuations in two dimensions. Ann. Prob. 23, 977-1005. 
[16] Pimentel, L. P. R. (2004). Competing growth, interfaces and geodesics in first-passage percolation on Voronoi tilings. Doctoral Thesis, IMPA, Rio de Janeiro.

[17] Pimentel, L. P. R. (2005). Asymptotics for first-passage times on Delaunay triangulations. Submitted.

[18] Pimentel, L. P. R. (2006). The time constant and critical probabilities for percolation models. Electron. Commun. Prob. 11, 160-167.

[19] Saito, Y. and Müller-Krumbhaar, M. (1995). Critical phenomena in morphology transitions of growth models with competition. Phys. Rev. Lett. 74, 4325-4328.

[20] Vahidi-Asl, M. Q. AND Wierman, J. C. (1990). First-passage percolation on the Voronoi tessellation and Delaunay triangulation. In Random Graphs '87, eds M. Karonske, J. Jaworski and A. Rucinski, John Wiley, Chichester, pp. 341-359.

[21] VAhidi-AsL, M. Q. ANd Wierman, J. C. (1992). A shape result for first-passage percolation on the Voronoi tessellation and Delaunay triangulation. In Random Graphs '89, eds A. Frieze and T. Luczak, John Wiley, Chichester, pp. 247-262. 